Physics

(TID-4500, 33rd Ed.)

AEC Research and

Development Report

ARGONNE NATIONAL LABORATORY

9700 South Cass Avenue

Argonne, Illinois 60440

\title{
BENDING OF CIRCULAR PLATES \\ UNDER A UNIFORM LOAD \\ ON A CONCENTRIC CIRCLE
}

$$
\text { by }
$$

J. C. Heap

Particle Accelerator Division

April 1964

Operated by The University of Chicago under

Contract W-31-109-eng-38

with the

U. S. Atomic Energy Commission 


\section{DISCLAIMER}

This report was prepared as an account of work sponsored by an agency of the United States Government. Neither the United States Government nor any agency Thereof, nor any of their employees, makes any warranty, express or implied, or assumes any legal liability or responsibility for the accuracy, completeness, or usefulness of any information, apparatus, product, or process disclosed, or represents that its use would not infringe privately owned rights. Reference herein to any specific commercial product, process, or service by trade name, trademark, manufacturer, or otherwise does not necessarily constitute or imply its endorsement, recommendation, or favoring by the United States Government or any agency thereof. The views and opinions of authors expressed herein do not necessarily state or reflect those of the United States Government or any agency thereof. 


\section{DISCLAIMER}

Portions of this document may be illegible in electronic image products. Images are produced from the best available original document. 


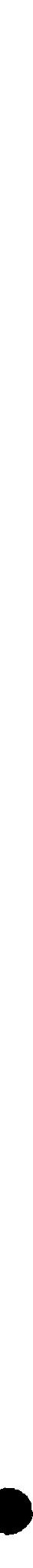




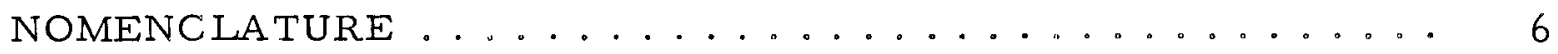

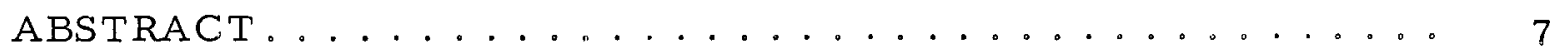

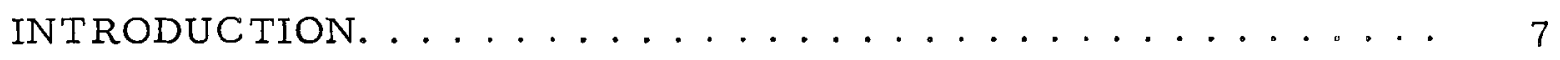

SYSTEM OF UNITS. .......................... 8

THEORETICAL DEVELOPMENT ................. 9

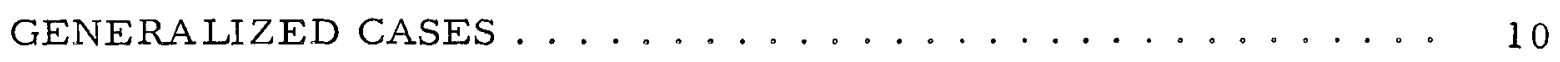

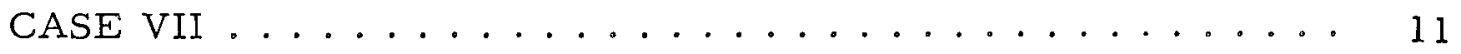

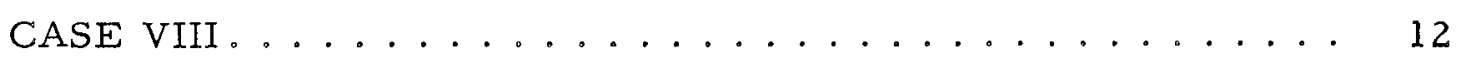

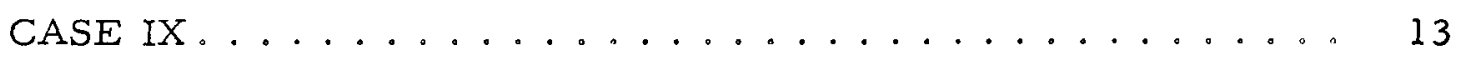

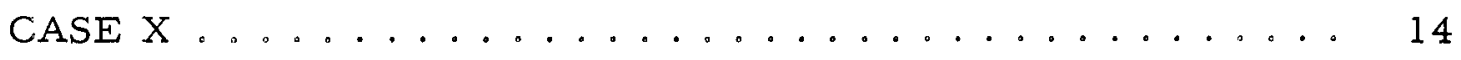

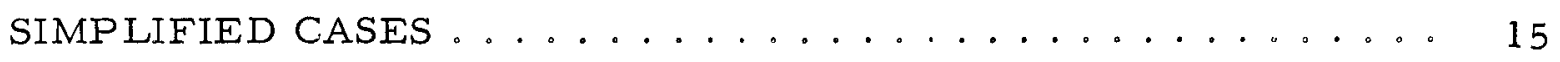

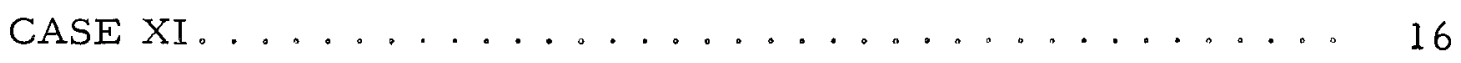

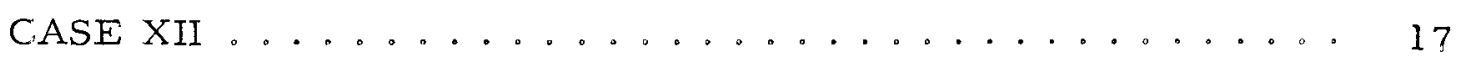

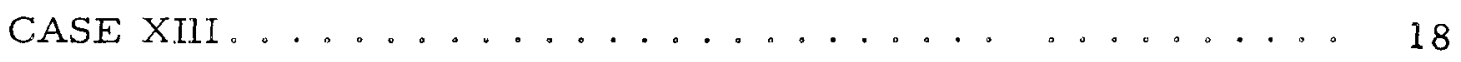

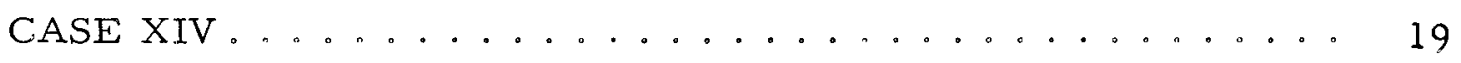

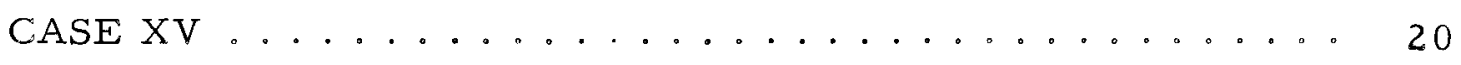

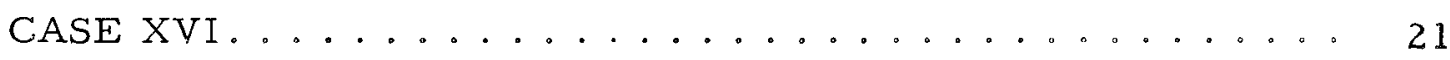

DESIGN CONSIDERATIONS ................... 22

STATICALLY INDETERMINATE CIRCULAR PLATES . . . . . 36

DISCUSSION OF GENERA LIZED CASES . . . . . . . . . . 40

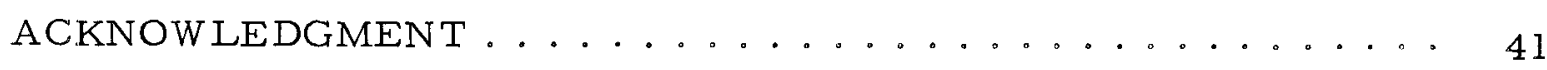

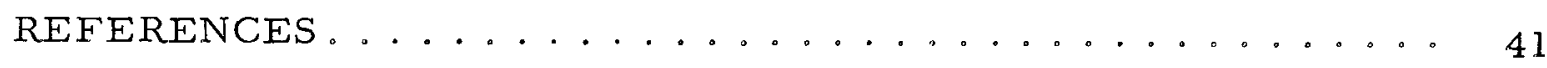




\section{LIST OF FIGURES}

No.

$\underline{\text { Title }}$

$\underline{\text { Page }}$

1. Uniform Load Acting on a Concentric Circle of a Thin,

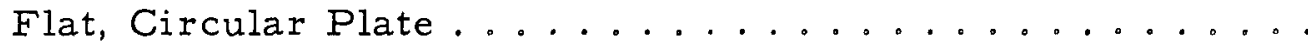

2. Radial and Tangential Moments per Uniform Load on Concentric Circle for Circular Plate Having Fixed Supported Outer Edge and Fixed Inner Edge (Case VII, $\nu=0.3$ )....

3. Radial and Tangential Moments per Uniform Load on Concentric Circle for Circular Plate Having Simply Sup ported Outer Edge and Free Inner Edge (Case VIII, $\nu=0.3$ ). 。

4. Radial and Tangential Moments per Uniform Load on Concentric Circle for Circular Plate Having Simply Supported Outer Edge and Fixed Inner Edge (Case IX, $\nu=0.3$ ) .

5. Radial and Tangential Moments per Uniform Load on Concentric Circle for Circular Plate Having Fixed Supported Outer Edge and Free Inner Edge (Case X, $v=0.3$ ) 。

6. Radial and Tangential Moments per Uniform Load on Inner Concentric Circle for Circular Plate Having Fixed Supported Outer Edge and Fixed Inner Edge (Case XI, $y=0.3$ ).

7. Radial and Tangential Moments per Uniform Load on Inner Concentric Circle for Circular Plate Having Simply Supported Outer Edge and Free Inner Edge (Case XII, $\nu=0.3$ ). .

8. Radial and Tangential Moments per Uniform Load on Inner Concentric Circle for Circular Plate Having Simply Supported Outer Edge and Fixed Inner Edge (Case XIII, $v=0.3$ ).

9. Radial and Tangential Moments per Uniform Load on Inner Concentric Circle for Circular Plate Having Fixed Supported Outer Edge and Free Inner Edge (Case XIV, $\nu=0.3$ ).

10. Radial and Tangential Moments per Uniform Load on Concentric Circle for Solid Circular Plate Having Fixed

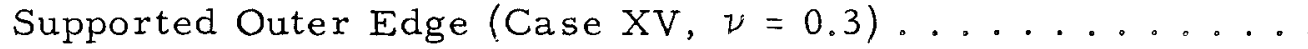

11. Radial and Tangential Moments per Uniform Load on Concentric Circle for Solid Circular Plate Having Simply

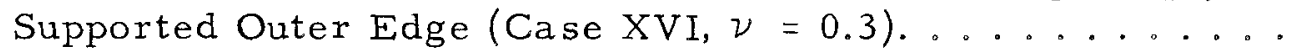

12. Statically Indeterminate Diaphragm Subjected to a Symmetrical Variable Load ..................

13. Statıcally Indeterminate Circular Plate Subjected to Symmetrical Variable Load and Uniform Inner Edge Load. . . . . 


\section{LIST OF TABLES}

No.

Title

$\underline{\text { Page }}$

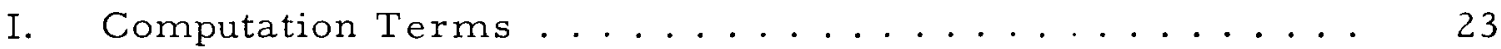

II. Maximum Deflection and Moment Constants Where

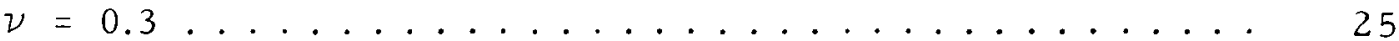


NOMENCLATURE

English

Letters

a

b

Outer plate support radius

Radius of uniform load on inner

concentric circle and/or inner

plate radius

d

Radius of uniform load on con-

centric circle

$\mathrm{C}_{1}$

$\mathrm{C}_{2}$

$\mathrm{C}_{3}$

$\mathrm{C}_{4}$

$\mathrm{C}_{5}$

$\mathrm{C}_{6}$

D

E

E

h

k

$\mathrm{k}_{\mathrm{d}}$

$\mathrm{k}_{\mathrm{m}}$

$\mathrm{k}_{\mathrm{r}}$

$\mathrm{kt}$

$M_{\max }$

$\mathrm{M}_{\mathrm{r}}$

$M_{\text {ra }}$

$\mathrm{M}_{\mathrm{rb}}$

$M_{t}$

Constants of integration for outer portion of plate, bounded by uniform load on concentric circle and outer plate support radius.

Constants of integration for inner portion of plate, bounded by uniform load on concentric circle and inner plate radius

Flexural rigidity of plate, symbolically $\mathrm{Eh}^{3} / 12\left(1-\nu^{2}\right)$

Modulus of elasticity

Uniform plate thickness

Deflection constant

Maximum deflection constant

Maximum bending-moment constant

Radial bending-moment constant

Tangential bending-moment constant

Maximum bending moment per unit length

Radial bending moment per unit length

Radial bending moment per unit length at outer plate support radius

Radial bending moment per unit length at inner plate radius

$M_{t} \quad$ Tangential bending moment per unit

\begin{tabular}{lll}
\multicolumn{1}{c}{$\begin{array}{l}\text { British } \\
\text { in. }\end{array}$} & \multicolumn{1}{c}{$\begin{array}{c}\text { Units } \\
\mathrm{cm}\end{array}$} \\
in. & $\mathrm{cm}$ \\
in. & & $\mathrm{cm}$ \\
1/in. & & $1 / \mathrm{cm}$ \\
in. & $\mathrm{cm}$ \\
in. & $\mathrm{cm}$ \\
1/in. & $1 / \mathrm{cm}$ \\
in. & $\mathrm{cm}$ \\
in. & $\mathrm{cm}$
\end{tabular}

$1 b_{f}-$ in. $\quad \mathrm{kg}_{f}-\mathrm{cm}$

$\mathrm{lb}_{\mathrm{f}} / \mathrm{in} .^{2} \quad \mathrm{~kg}_{\mathrm{f}} / \mathrm{cm}^{2}$

in. $\mathrm{cm}$ length

\footnotetext{
$1 b_{f}$-in./in. $\quad \mathrm{kg}_{f}-\mathrm{cm} / \mathrm{cm}$

$1 \mathrm{~b}_{f^{-i n}}$. in. $\quad \mathrm{kg}_{f}-\mathrm{cm} / \mathrm{cm}$

$1 b_{f}-i n . / i n . \quad \operatorname{kg}_{f}-\mathrm{cm} / \mathrm{cm}$

$1 b_{f}-i n . / i n . \quad \mathrm{kg}_{f}-\mathrm{cm} / \mathrm{cm}$

$\mathrm{lb}_{\mathrm{f}}-\mathrm{in} . / \mathrm{in} . \quad \mathrm{kg}_{\mathrm{f}}-\mathrm{cm} / \mathrm{cm}$
}

English

Letters

$\mathrm{M}_{\text {ta }}$

$\mathrm{M}_{\mathrm{tb}}$

$\mathrm{P}$

$\mathrm{R}$

r

$\mathrm{V}$

W

w

w

${ }^{\mathrm{w}} \mathrm{d}$

Greek

Letters

$\nu$

$\sigma \max$

$\sigma r$

$\sigma_{\mathrm{rb}}$

$\sigma_{\mathrm{t}}$

$\phi$
Description

Tangential bending moment per unit length at outer plate support radius

Tangential bending moment per unit length at inner plate radius

\section{Constant force}

Redundant load

Radius of plate

Shearing force per unit circum-

ferential length

Uniform load on a concentric circle of plate

Deflection of plate

Deflection of plate at uniform load on concentric circle

\section{Poisson's ratio}

Maximum unit stress

Radial unit stress

Tangential unit stress
Bending angle

\begin{tabular}{|c|c|}
\hline $\begin{array}{c}\text { British } \\
\text { Units } \\
\end{array}$ & $\begin{array}{c}\text { Metric } \\
\text { Units }\end{array}$ \\
\hline $1 b_{f}-i n . / i n$ & $\mathrm{~kg}_{\mathrm{f}}-\mathrm{cm} / \mathrm{cm}$ \\
\hline $1 b_{f}-i n . / i n$ & $\mathrm{~kg}_{\mathrm{f}}-\mathrm{cm} / \mathrm{cm}$ \\
\hline $\mathrm{lb}_{\mathrm{f}}$ & $\mathrm{kg}_{\mathrm{f}}$ \\
\hline $1 b_{f}$ & $\mathrm{~kg}_{\mathrm{f}}$ \\
\hline in. & $\mathrm{cm}$ \\
\hline $\mathrm{lb}_{\mathrm{f}} / \mathrm{in}$ & $\mathrm{kg}_{\mathrm{f}} / \mathrm{cm}$ \\
\hline $\mathrm{lb}_{\mathrm{f}}$ & $\mathrm{kg}_{\mathrm{f}}$ \\
\hline in. & $\mathrm{cm}$ \\
\hline in. & $\mathrm{cm}$ \\
\hline $\begin{array}{l}\text { British } \\
\text { Units }\end{array}$ & $\begin{array}{l}\text { Metric } \\
\text { Units }\end{array}$ \\
\hline
\end{tabular}

$\mathrm{lb}_{\mathrm{f}} / \mathrm{in}^{2} \quad \quad \mathrm{~kg}_{\mathrm{f}} / \mathrm{cm}^{2}$

$\mathrm{lb}_{\mathrm{f}} / \mathrm{in}^{2} \quad \mathrm{~kg} \mathrm{f} / \mathrm{cm}^{2}$

$\mathrm{lb}_{\mathrm{f}} / \mathrm{in} .^{2} \quad \mathrm{~kg}_{\mathrm{f}} / \mathrm{cm}^{2}$

$\mathrm{lb}_{\mathrm{f}} / \mathrm{in}^{2} \quad \mathrm{~kg}_{\mathrm{f}} / \mathrm{cm}^{2}$

$\mathrm{rad} \mathrm{rad}$ 


\title{
BENDING OF CIRCULAR PLATES \\ UNDER A UNIFORM LOAD \\ ON A CONCENTRIC CIRCLE
}

by

J. C. Heap

\begin{abstract}
The basic equations of deflection, slope, and moments for a thin, flat, circular plate subjected to a uniform load on a concentric circle were derived for four generalized cases. From these generalized cases, six simplified cases were deduced. The four generalized cases have the uniform load acting on a concentric circle of the plate between the inner and outer edges, with the following boundary conditions: (1) outer edge supported and fixed, inner edge fixed; (2) outer edge simply supported, inner edge free: (3) outer edge simply supported, inner edge fixed; and (4) outer edge supported and fixed, inner edge free.
\end{abstract}

\section{INTRODUCTION}

Analyses of thin, flat, circular plates subjected to bending were essential in the design and development of experimental equipment for the Argonne National Laboratory Zero Gradient Synchrotron. Plate thickness was minimized since the absorption of high-energy particles depends upon the thickness and material.

Because there were more supports than necessary to maintain stability of the plates, the solution of statically indeterminate plates was inevitable. Removal of redundant support would impair not only the structural integrity of the plates, but also that of affiliated components. Superposition is the usual procedure for solving statically indeterminate problems; however, frequently the generalized equations of deflection, moments, or slope must be known for the analysis.

This paper presents four generalized cases for a uniform load acting on a concentric circle of a thin, flat, circular plate (schematically depicted by Fig. 1), for solving statically indeterminate plates. The four generalized 
cases are: (1) outer edge supported and fixed, inner edge fixed; (2) outer edge simply supported, inner edge free: (3) outer edge simply supported, inner edge fixed; and (4) outer edge supported and fixed, inner edge free. From these four generalized cases, six simplified cases are derived. The first four simplified cases have the uniform load along the inner plate radius, and boundary conditions complying to the four generalized cases. The last two cases are for a solid plate where the outer edge conditions are fixed-supported and simply supported.

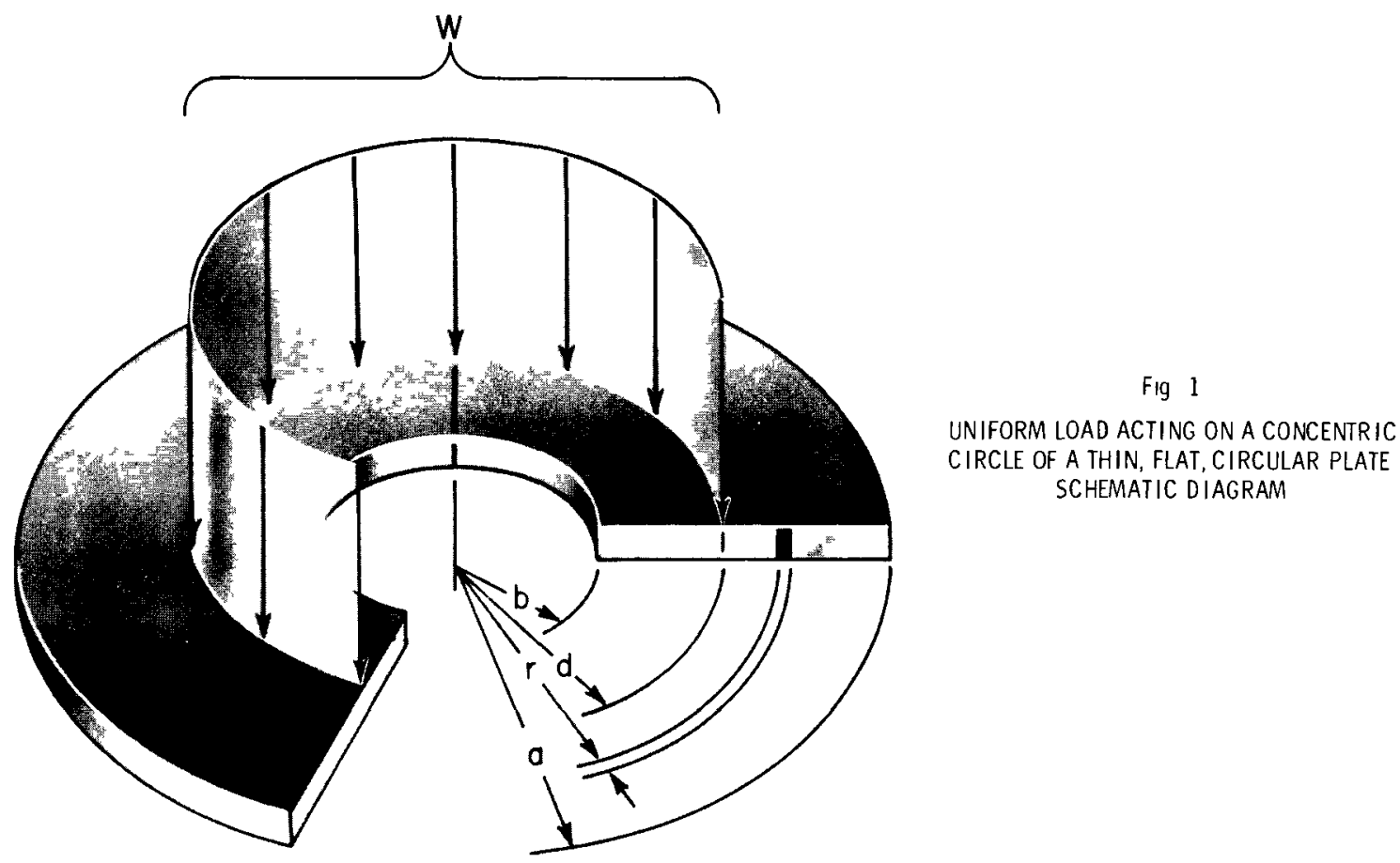

A computer program was developed for resolving deflections and moments of these ten cases and for assisting in the solution for statically indeterminate circular plates. When only one or two computations are required, various dimensionless terms in the developed equations have been computed and tabulated to simplify deflection, moment, and slope calculations.

The cases have been numbered VII through XVI since this is a continuation of the investigation initiated in Ref. 1. Moreover, this consecutive numbering of the cases eliminates confusion that could arise between the deflection-moment computer programs.

\section{SYSTEM OF UNITS}

In this presentation, the unit force-mass system is used since it provides a compromise between the absolute and gravitational systems and is automatically a self-containing reference system. A comprehensive analysis of this system is contained in Ref. 2 . 
From Ref. 1, 3, or 4, the fundamental equations for thin, flat, circular plates subjected to symmetrical loads are as follows: (1) The radial and tangential bending moments per unit length are

$$
\begin{aligned}
& M_{r}=D\left(\frac{d \phi}{d r}+\nu \frac{\phi}{r}\right) ; \\
& M_{t}=D\left(\frac{\phi}{r}+\nu \frac{d \phi}{d r}\right) ;
\end{aligned}
$$

and (2) the equilibrium equation is

$$
\frac{\mathrm{d}^{2} \phi}{\mathrm{d} \mathrm{r}^{2}}+\frac{1}{\mathrm{r}} \frac{\mathrm{d} \phi}{\mathrm{dr}}-\frac{\phi}{\mathrm{r}^{2}} \equiv \frac{\mathrm{d}}{\mathrm{dr}}\left[\frac{1}{\mathrm{r}} \frac{\mathrm{d}}{\mathrm{dr}}(\mathrm{r} \phi)\right]=-\frac{\mathrm{V}}{\mathrm{D}},
$$

where $\phi=-\mathrm{dw} / \mathrm{dr}$ and $\mathrm{D}=\mathrm{Eh}^{3} / 12\left(1-\nu^{2}\right)$.

From Fig. 1, the shearing force per unit tangential length at any radius for the outer portion is

$$
V=\frac{W}{2 \pi r},
$$

and for the inner portion is

$$
\mathrm{V}=0 \text {. }
$$

Substituting these shearing forces into the equilibrium equation, integrating thrice, and taking the derivation of the $\phi$ expression, one obtains, for the outer portion of the plate, $d \leq r \leq a$,

$$
\begin{aligned}
-\frac{\mathrm{dw}}{\mathrm{dr}} & =\phi=-\frac{\mathrm{W}}{4 \pi \mathrm{D}} \mathrm{r}\left(\ln r-\frac{1}{2}\right)+\frac{\mathrm{C}_{1}}{2} \mathrm{r}+\frac{\mathrm{C}_{2}}{\mathrm{r}} \\
\frac{\mathrm{d} \phi}{\mathrm{dr}} & =-\frac{\mathrm{W}}{4 \pi \mathrm{D}}\left(\ln \mathrm{r}+\frac{1}{2}\right)+\frac{\mathrm{C}_{1}}{2}-\frac{\mathrm{C}_{2}}{\mathrm{r}^{2}} ; \\
\mathrm{w} & =\frac{\mathrm{W}}{8 \pi \mathrm{D}} \mathrm{r}^{2}(\ln \mathrm{r}-1)-\frac{\mathrm{C}_{1}}{4} \mathrm{r}^{2}-\mathrm{C}_{2} \ln \mathrm{r}+\mathrm{C}_{3} ;
\end{aligned}
$$

and for the inner portion of the plate, $b \leq r \leq d$, 


$$
\begin{aligned}
-\frac{\mathrm{dw}}{\mathrm{dr}} & =\phi=\frac{\mathrm{C}_{4}}{2} r+\frac{\mathrm{C}_{5}}{\mathrm{r}} \\
\frac{\mathrm{d} \phi}{\mathrm{dr}} & =\frac{\mathrm{C}_{4}}{2}-\frac{\mathrm{C}_{5}}{\mathrm{r}^{2}} \\
\mathrm{w} & =-\frac{\mathrm{C}_{4}}{4} \mathrm{r}^{2}-\mathrm{C}_{5} \ln \mathrm{r}+\mathrm{C}_{6}
\end{aligned}
$$

Upon substitution of the appropriate portions of Eqs. (5) and (6) into Eq. (1), the moment equations become, for the outer portion,

$$
\begin{aligned}
& M_{r}=-\frac{W}{4 \pi}\left[(1+\nu) \ln r+\frac{1}{2}(1-\nu)\right]+D\left[\frac{C_{1}}{2}(1+\nu)-\frac{C_{2}}{r^{2}}(1-\nu)\right] \\
& M_{t}=-\frac{W}{4 \pi}\left[(1+\nu) \ln r-\frac{1}{2}(1-\nu)\right]+D\left[\frac{C_{1}}{2}(1+\nu)+\frac{C_{2}}{r^{2}}(1-\nu)\right]
\end{aligned}
$$

and for the inner portion,

$$
\begin{aligned}
& M_{r}=D\left[\frac{C_{4}}{2}(1+\nu)-\frac{C_{5}}{r^{2}}(1-\nu)\right] \\
& M_{t}=D\left[\frac{C_{4}}{2}(1+\nu)+\frac{C_{5}}{r^{2}}(1-\nu)\right] .
\end{aligned}
$$

\section{GENERALIZED CASES}

The four generalized cases presented in tabular form (Cases VII through $\mathrm{X}$ ) were derived by applying the appropriate expressions that fulfill the continulty and boundary conditions. The integration constants were determined from the first and third expressions of Eqs. (5) and (6), and from the expression of Eqs. (7) and (8). The upperright corner of each tabulated case shows the continuity conditions and/or boundary conditions. 
GASE VII

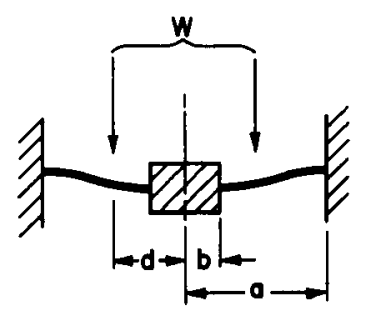

\section{Description}

Outer edge supported and fixed. Inner edge fixed.

Uniform load on a concentric circle of plato.
Boundary Conditiong

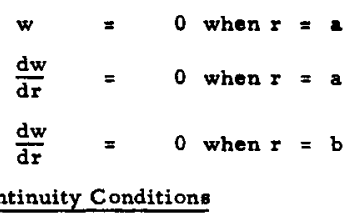

Continuity Conditions when $r=d$

inner portion of plate

same as outer portion

Inner portion of plate, $b \leq r \leq d$

$$
\begin{aligned}
& M_{r}=\frac{w}{4 \pi}(1+v)\left(\frac{a^{2}}{a^{2}-b^{2}}\right)\left[\ln \frac{a}{d}-\frac{1}{2}\left(1-\frac{d^{2}}{a^{2}}\right)\right]\left[1+\left(\frac{1-v}{1+v}\right) \frac{b^{2}}{r^{2}}\right] \\
& M_{r b}=\frac{W}{2 \pi}\left(\frac{a^{2}}{a^{2}-b^{2}}\right)\left[\ln \frac{a}{d}-\frac{1}{2}\left(1-\frac{d^{2}}{a^{2}}\right)\right] \\
& M_{t}=\frac{w}{4 \pi}(1+v)\left(\frac{a^{2}}{a^{2}-b^{2}}\right)\left[\ln \frac{a}{d}-\frac{1}{2}\left(1-\frac{d^{2}}{2}\right)\right]\left[1-\left(\frac{1-v}{1+v}\right) \frac{b^{2}}{x^{2}}\right] \\
& \mathrm{M}_{\mathrm{tb}}=\nu \mathrm{M}_{\mathbf{x b}}
\end{aligned}
$$

Slope

$$
\frac{d w}{d r}=-\frac{W r}{4 \pi D}\left(\frac{a^{2}}{a^{2}-b^{2}}\right)\left[\ln \frac{a}{d}-\frac{1}{2}\left(1-\frac{d^{2}}{a^{2}}\right)\right]\left(1-\frac{b^{2}}{r^{2}}\right)
$$

$$
\begin{aligned}
& \text { Doflection } \\
& w=\frac{W a^{2}}{8 \pi D}\left(\frac{a^{2}}{a^{2}-b^{2}}\right)\left\{\left(1-\frac{d^{2}}{a^{2}}\right)\left[\left(1-\frac{b^{2}}{a^{2}}\right)-\frac{1}{2}\left(1-\frac{x^{2}}{a^{2}}\right)\right]+\frac{b^{2}}{a}\left(1-\frac{a^{2}}{a}\right) \ln \frac{d}{x}+\left(2 \frac{b^{2}}{a^{2}}-\frac{d^{2}}{a^{2}}-\frac{r^{2}}{a}\right) \ln \frac{a}{d}-2 \frac{b^{2}}{a}\left(\ln \frac{a}{d}\right) \ln \frac{a}{r}\right\} \\
& w_{\max }=\frac{W a^{2}}{8 \pi D}\left[\frac{1}{2}\left(1-\frac{d^{2}}{a^{2}}\right)+\frac{b^{2}}{a^{2}-b^{2}} \ln \frac{a}{b}-\frac{d^{2}}{a^{2}-b^{2}}\left(\frac{b^{2}}{a^{2}} \ln \frac{d}{b}+\ln \frac{a}{d}\right)-\frac{2 b^{2}}{a^{2}-b^{2}}\left(\ln \frac{a}{b}\right) \ln \frac{a}{d}\right]
\end{aligned}
$$

Outer portion of plate, $d \leq x \leq a$

$$
\begin{aligned}
& M_{r}=\frac{W}{4 \pi}(1+v)\left(\frac{a^{2}}{a^{2}-b^{2}}\right)\left\{\frac{1}{2}\left(\frac{1-v}{1+v}\right)\left[\frac{b^{2}}{a^{2}}-\frac{b^{2}}{r^{2}}-\left(1-\frac{d^{2}}{r^{2}}\right)\right]-\frac{1}{2}\left(1-\frac{d^{2}}{a^{2}}\right)+\ln \frac{a}{r}+\frac{b^{2}}{a^{2}} \ln \frac{r}{d}+\left(\frac{1-v}{1+v}\right) \frac{b^{2}}{r^{2}} \ln \frac{a}{d}\right\} \\
& M_{r a}=\frac{w}{2 \pi}\left(\frac{a^{2}}{a^{2}-b^{2}}\right)\left[\frac{b^{2}}{a^{2}} \ln \frac{a}{d}-\frac{1}{2}\left(1-\frac{d^{2}}{a^{2}}\right)\right. \\
& M_{t}=\frac{W}{4 \pi}(1+v)\left(\frac{a^{2}}{a^{2}-b^{2}}\right)\left\{\frac{1}{2}\left(\frac{1-v}{1+v}\right)\left[\frac{b^{2}}{x^{2}}-\frac{b^{2}}{a^{2}}+\left(1-\frac{d^{2}}{x^{2}}\right)\right]-\frac{b}{2}\left(1-\frac{a^{2}}{a^{2}}\right)+\ln \frac{a}{x}+\frac{b^{2}}{a^{2}} \ln \frac{x}{d}-\left(\frac{1-v}{1+v}\right) \frac{b^{2}}{x^{2}} \ln \frac{a}{d}\right\} \\
& M_{\text {ta }}=v M_{r a} \\
& \frac{d w}{d r}=-\frac{W r}{4 \pi D}\left(\frac{a^{2}}{a^{2}-b^{2}}\right)\left[\ln \frac{a}{r}+\frac{b^{2}}{a^{2}} \ln \frac{x}{d}-\frac{b^{2}}{x^{2}} \ln \frac{a}{d}-\frac{1}{2}\left(1-\frac{r^{2}}{a^{2}}\right)\left(\frac{d^{2}}{r^{2}}-\frac{b^{2}}{r^{2}}\right)\right] \\
& w=\frac{W a^{2}}{8 \pi D}\left(\frac{a^{2}}{a^{2}-b^{2}}\right)\left\{\left(1-\frac{x^{2}}{a^{2}}\right)\left[\left(1-\frac{b^{2}}{a^{2}}\right)-\frac{1}{2}\left(1-\frac{d^{2}}{a^{2}}\right)\right]+\frac{b^{2}}{a^{2}}\left(1-\frac{x^{2}}{a^{2}}\right) \ln \frac{r}{d}+\left(2 \frac{b^{2}}{a^{2}}-\frac{d^{2}}{a^{2}}-\frac{x^{2}}{a^{2}}\right) \ln \frac{a}{r}-2 \frac{b^{2}}{a^{2}}\left(\ln \frac{a}{d}\right) \ln \frac{a}{r}\right\}
\end{aligned}
$$


CASE VIII

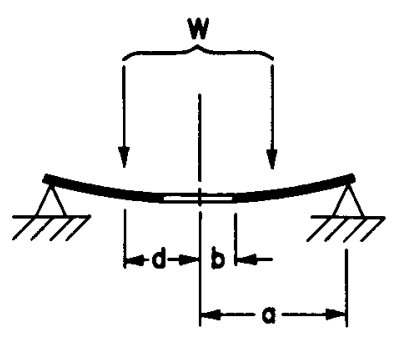

Description

Outer edge simply supported.

Inner edge free.

Uniform load on a concentric cricle of plate.
Boundary Conditions

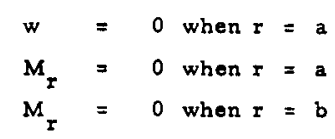

Continuity Conditions

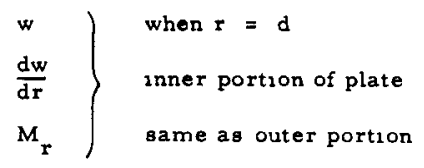

Inner portion of plate, $b \leq r \leq d$

Moments

$$
\begin{aligned}
& M_{r}=\frac{w}{4 \pi}(1+v)\left(1-\frac{b^{2}}{r^{2}}\right)\left(\frac{a^{2}}{a^{2}-b^{2}}\right)\left[\frac{1}{2}\left(\frac{1-v}{1+v}\right)\left(1-\frac{d^{2}}{a^{2}}\right)+\ln \frac{a}{d}\right] \\
& M_{t}=\frac{W}{4 \pi}(1+v)\left(1+\frac{b^{2}}{r^{2}}\right)\left(\frac{a^{2}}{a^{2}-b^{2}}\right)\left[\frac{1}{2}\left(\frac{1-v}{1+v}\right)\left(1-\frac{d^{2}}{a^{2}}\right)+\ln \frac{a}{d}\right] \\
& M_{\max }=M_{t b}=\frac{w}{2 \pi}(1+v)\left(\frac{a^{2}}{a^{2}-b^{2}}\right)\left[\frac{1}{2}\left(\frac{1-v}{1+v}\right)\left(1-\frac{d^{2}}{a^{2}}\right)+\ln \frac{a}{d}\right]
\end{aligned}
$$

Slope

$$
\frac{d w}{d r}=-\frac{w r}{4 \pi D}\left(\frac{a^{2}}{a^{2}-b^{2}}\right)\left[1+\left(\frac{1+v}{1-v}\right) \frac{b^{2}}{r^{2}}\right]\left[\frac{1}{2}\left(\frac{1-v}{1+v}\right)\left(1-\frac{d^{2}}{a^{2}}\right)+\ln \frac{a}{d}\right]
$$

$$
\begin{aligned}
& w=\frac{w a^{2}}{8 \pi D}\left(\frac{a^{2}}{a^{2}-b^{2}}\right)\left\{\left(1-\frac{d^{2}}{a^{2}}\right)\left[\left(1-\frac{b^{2}}{a^{2}}\right)+\frac{1}{2}\left(\frac{1-v}{l+v}\right)\left(1-\frac{r^{2}}{a^{2}}\right)\right]+\frac{b^{2}}{a^{2}}\left(1-\frac{a^{2}}{a^{2}}\right) \ln \frac{d}{r}+\left(2 \frac{b^{2}}{a^{2}}-\frac{r^{2}}{a^{2}}-\frac{d^{2}}{a^{2}}\right) \ln \frac{a}{d}+2\left(\frac{1+v}{1-v}\right)\left(\frac{b^{2}}{a^{2}}\right)\left(\ln \frac{a}{d}\right) \ln \frac{a}{r}\right\rangle \\
& w_{\max }=\frac{w^{2}}{8 \pi D}\left(\frac{1}{2}\left(\frac{3+v}{1+v}\right)\left(1-\frac{d^{2}}{a^{2}}\right)+\left(\frac{b^{2}}{a^{2}-b^{2}}\right) \ln \frac{a}{b}-\frac{d^{2}}{a^{2}-b^{2}}\left(\frac{b^{2}}{a^{2}} \ln \frac{d}{b}+\ln \frac{a}{d}\right)+2\left(\frac{1+v}{1-v}\right)\left(\frac{b^{2}}{a^{2}-b^{2}}\right)\left(\ln \frac{a}{b}\right) \ln \frac{a}{d}\right)
\end{aligned}
$$

Outer portion of plate, $d \leq r \leq a$

\section{Moments}

$$
\begin{aligned}
& M_{r}=\frac{w}{4 \pi}(1+v)\left(\frac{a^{2}}{a^{2}-b^{2}}\right)\left[\frac{l}{2}\left(\frac{l-v}{1+v}\right)\left(1-\frac{r^{2}}{a^{2}}\right)\left(\frac{d^{2}}{r^{2}}-\frac{b^{2}}{r^{2}}\right)+\ln \frac{a}{r}+\frac{b^{2}}{a^{2}} \ln \frac{r}{d}-\frac{b^{2}}{r^{2}} \ln \frac{a}{d}\right] \\
& M_{t}=\frac{W}{4 \pi}(1+v)\left(\frac{a^{2}}{a^{2}-b^{2}}\right)\left\{\frac{1}{2}\left(\frac{1-v}{1+v}\right)\left[\left(1-\frac{d^{2}}{a^{2}}\right)\left(1+\frac{a^{2}}{r^{2}}\right)-\left(1-\frac{r^{2}}{a^{2}}\right)\left(\frac{a^{2}}{r^{2}}-\frac{b^{2}}{r^{2}}\right)\right]+\ln \frac{a}{r}+\frac{b^{2}}{a^{2}} \ln \frac{r}{d}+\frac{b^{2}}{r^{2}} \ln \frac{a}{d}\right\} \\
& M_{t a}=\frac{W}{2 \pi}(1+v)\left(\frac{a^{2}}{a^{2}-b^{2}}\right)\left[\frac{1}{2}\left(\frac{1-v}{1+v}\right)\left(1-\frac{d^{2}}{a^{2}}\right)+\frac{b^{2}}{a^{2}} \ln \frac{a}{d}\right] \\
& \frac{d w}{d r}=-\frac{w r}{4 \pi D}\left(\frac{a^{2}}{a^{2}-b^{2}}\right)\left\{\frac{1}{2}\left[\left(1-\frac{a^{2}}{z^{2}}\right)\left(1-\frac{b^{2}}{a^{2}}\right)+\left(1-\frac{a^{2}}{a^{2}}\right)\left(\frac{a^{2}}{r^{2}}+\frac{1-v}{1+v}\right)\right]+\ln \frac{a}{r}+\frac{b^{2}}{a^{2}} \ln \frac{r}{d}+\left(\frac{1+v}{1-v}\right) \frac{b^{2}}{r^{2}} \ln \frac{a}{d}\right\}
\end{aligned}
$$

Deflection

$$
w=\frac{w a^{2}}{8 \pi D}\left(\frac{a^{2}}{a^{2}-b^{2}}\right)\left\{\left(1-\frac{r^{2}}{a^{2}}\right)\left[\left(1-\frac{b^{2}}{a^{2}}\right)+\frac{1}{2}\left(\frac{1-v}{1+v}\right)\left(1-\frac{d^{2}}{a^{2}}\right)\right]+\left(\frac{b^{2}}{a^{2}}-\frac{d^{2}}{a^{2}}-\frac{r^{2}}{a^{2}}\right) \ln \frac{a}{r}+\frac{b^{2}}{a^{2}}\left(\ln \frac{a}{d}-\frac{r^{2}}{a^{2}} \ln \frac{r}{d}\right)+2\left(\frac{l+v}{1-\nu}\right)\left(\frac{b^{2}}{a^{2}}\right)\left(\ln \frac{a}{r}\right) \ln \frac{a}{d}\right\rangle
$$


CASE IX

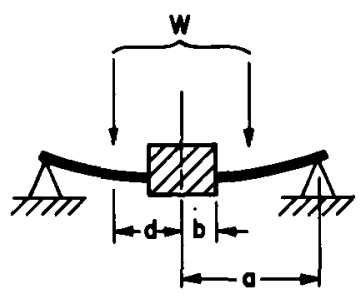

\section{Description}

Outer edge aimply oupported. Inner odge fixed.

Uniform load on a concentric circle of plate.
Boundaxy Conditions

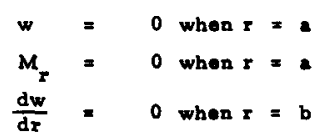

Continuity Condition:

$w$
$\frac{d w}{d x}$
$M$$\left\{\begin{array}{l}\text { when } r=d \\ \text { inner portion of plate }\end{array}\right.$

Inner portion of plate, $b=x=d$

$$
\begin{aligned}
& M_{T}=\frac{W}{4 \pi}(l-v)\left[\frac{\frac{1+v}{1-v}+\frac{b^{2}}{r^{2}}}{\frac{l+v}{l-v}+\frac{b^{2}}{a^{2}}}\right]\left[\frac{1}{2}\left(1-\frac{d^{2}}{a^{2}}\right)+\left(\frac{1+v}{1-v}\right) \ln \frac{a}{d}\right] \\
& M_{r b}=\frac{w}{2 \pi}\left[\frac{1}{\frac{1+v}{1-v}+\frac{b^{2}}{a^{2}}}\right]\left[\frac{1}{2}\left(1-\frac{d^{2}}{a^{2}}\right)+\left(\frac{1+v}{1-v}\right) \ln \frac{a}{d}\right] \\
& M_{t}=\frac{W}{4 \pi}(1-v)\left[\frac{\frac{1+v}{1-v}-\frac{b^{2}}{c^{2}}}{\frac{1+v}{1-v}+\frac{b^{2}}{a^{2}}}\right]\left[\frac{1}{2}\left(1-\frac{d^{2}}{a^{2}}\right)+\left(\frac{1+v}{1-v}\right) \ln \frac{a}{d}\right] \\
& M_{t b}=v M_{r b}
\end{aligned}
$$

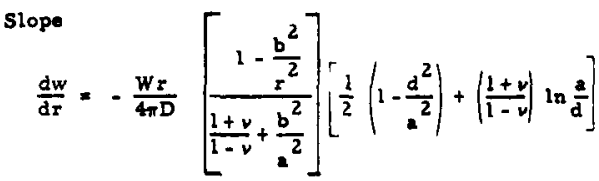

$$
\begin{aligned}
& \left.w=\frac{w a^{2}}{8 \pi D}\left[\frac{1}{\frac{l+v}{l-v}+\frac{b^{2}}{a^{2}}}\right]\left\{\frac{1}{2}\left(1-\frac{d^{2}}{a^{2}}\right)\left(\frac{3+v}{1-v}+2 \frac{b^{2}}{a^{2}}-\frac{r^{2}}{a^{2}}\right)-\left(1-\frac{d^{2}}{a^{2}}\right) \frac{b^{2}}{a^{2}} \ln \frac{d}{r}-\left[\left(\frac{l+v}{1-v}\right)\left(\frac{d^{2}}{a^{2}}+\frac{r^{2}}{a^{2}}\right)+2 \frac{b^{2}}{a^{2}}\right] \ln \frac{a}{d}-2\left(\frac{l+v}{l-v}\right)\left(\frac{b^{2}}{a^{2}}\right) \mid \ln \frac{a^{2}}{r}\right) \ln \frac{a}{d}\right\} \\
& w_{\max }=\frac{W_{a}^{2}}{8 \pi D}\left[\frac{1}{\frac{1+v}{1-v}+\frac{b^{2}}{a^{2}}}\right]\left\{\frac{1}{2}\left(1-\frac{d^{2}}{a^{2}}\right)\left(\frac{3+v}{b-v}+\frac{b^{2}}{a^{2}}\right)-\left(1-\frac{d^{2}}{a^{2}}\right) \frac{b^{2}}{a^{2}} \ln \frac{d}{b}-\left[\left(\frac{1+v}{1-v}\right) \frac{d^{2}}{a^{2}}+\left(\frac{3-v}{1-v}\right) \frac{b^{2}}{a^{2}}\right] \ln \frac{a}{d}-2\left(\frac{1+v}{1-v}\right)\left(\frac{b^{2}}{a^{2}}\right)\left(\ln \frac{a}{b}\right) \ln \frac{a}{d}\right\rangle
\end{aligned}
$$

Outer portion of plate, $d \leq x \leq a$

$$
\begin{aligned}
& M_{r}=\frac{w}{4 \pi}\left[\frac{1+v}{\frac{1+v}{1-v}+\frac{b^{2}}{a^{2}}}\right]\left\{\frac{1}{2}\left(1+\frac{r^{2}}{a^{2}}\right)\left[\frac{d^{2}}{x^{2}}+\left(\frac{1-v}{1+v}\right) \frac{b^{2}}{r^{2}}\right]+\frac{b^{2}}{r^{2}} \ln \frac{a}{d}+\left(\frac{1+v}{1-v}\right) \ln \frac{a}{r}-\frac{b^{2}}{a^{2}} \ln \frac{r}{d}\right\} \\
& M_{t}=\frac{W}{4 \pi}\left[\frac{1+v}{\frac{1+v}{1-v}+\frac{b^{2}}{a^{2}}}\right]\left\{\frac{1}{2}\left[\left(1-\frac{d^{2}}{a^{2}}\right)+\left(1-\frac{d^{2}}{x^{2}}\right)+\left(\frac{1-v}{1+v}\right)\left(\frac{b^{2}}{a^{2}}-\frac{b^{2}}{x^{2}}\right)\right]-\frac{b^{2}}{x^{2}} \ln \frac{a}{d}+\left(\frac{1+v}{1-v}\right) \ln \frac{a}{x}-\frac{b^{2}}{a^{2}} \ln \frac{x}{d}\right\} \\
& M_{t a}=\frac{w}{2 \pi}\left[\frac{1+v}{\frac{1+v}{1-v}+\frac{b^{2}}{a^{2}}}\right]\left[\frac{1}{2}\left(1-\frac{d^{2}}{a^{2}}\right)-\frac{b^{2}}{a^{2}} \ln \frac{a}{d}\right]
\end{aligned}
$$

slope

$$
\frac{d w}{d r}=-\frac{W x}{4 \pi D}\left[\frac{1}{\frac{1+v}{1-v}+\frac{b^{2}}{a^{2}}}\right]\left\{\frac{1}{2}\left[\left(\frac{1+v}{1-v}\right)\left(1-\frac{d^{2}}{r^{2}}\right)+\left(1-\frac{d^{2}}{a^{2}}\right)-\frac{b^{2}}{x^{2}}+\frac{b^{2}}{a^{2}}\right]-\left(\frac{1+v}{1-v}\right) \frac{b^{2}}{r^{2}} \ln \frac{a}{d}+\left(\frac{1+v}{1-\nu}\right) \ln \frac{a}{x}-\frac{b^{2}}{a^{2}} \ln \frac{r}{d}\right\}
$$

Deflection

$$
w=\frac{w a^{2}}{8 \pi D}\left[\frac{l}{\frac{1+v}{l-v}+\frac{b^{2}}{a^{2}}}\right]\left\{\frac{1}{2}\left(1-\frac{r^{2}}{a^{2}}\right)\left(\frac{3+v}{1-v}+2 \frac{b^{2}}{a^{2}}-\frac{d^{2}}{a^{2}}\right)-\left[\left(\frac{1+v}{1-v}\right)\left(\frac{d^{2}}{a^{2}}+\frac{r^{2}}{a^{2}}\right)+\frac{b^{2}}{a^{2}}\right] \ln \frac{a}{r}-\frac{b^{2}}{a^{2}} \ln \frac{a}{d}+\frac{r^{2}}{a^{2}}\left(\frac{b^{2}}{a^{2}}\right) \ln \frac{r}{d}-2\left(\frac{1+v}{i-v}\right)\left(\frac{b^{2}}{a^{2}}\right)\left(\ln \frac{a}{r}\right) \ln \frac{a}{d}\right]
$$


CASE $X$

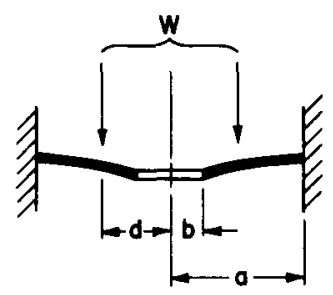

Deecription

Outor edse supported and fixed.

Inner edge tree.

Untform load on a concontric circle of plate.
Boundery Conditions

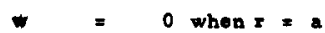

$\frac{d w}{d x} \quad * \quad 0$ when $r=a$

$\frac{d}{d x}$
$M_{\tau}$$\quad 0$ when $r=b$

Centinulty Conditions

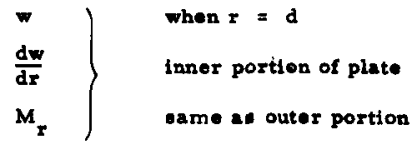

Inner portion of plate, $b \leq r \leq d$

Moments

$$
\begin{aligned}
& M_{r}=\frac{w}{4 \pi}\left[\frac{1-v}{\frac{1-v}{1+v}+\frac{b^{2}}{a^{2}}}\right]\left[-\frac{1}{2}\left(1-\frac{a^{2}}{a^{2}}\right)+\ln \frac{a}{d}\right]\left(1-\frac{b^{2}}{r^{2}}\right) \\
& \left.M_{t}=\frac{w}{4 \pi}\left[\frac{1-v}{\left.\frac{1-v}{1+v}+\frac{b^{2}}{a^{2}}\right]}\right]-\frac{1}{2}\left(1-\frac{d^{2}}{a^{2}}\right)+\ln \frac{a}{d}\right]\left(1+\frac{b^{2}}{r^{2}}\right) \\
& M_{t b}=\frac{w}{2 \pi}\left[\frac{1-v}{1-v}+\frac{b^{2}}{a^{2}}\right]\left[-\frac{1}{2}\left(1-\frac{d^{2}}{a^{2}}\right)+\ln \frac{a}{d}\right]
\end{aligned}
$$

$$
\begin{aligned}
& \text { Slope } \\
& \qquad \frac{d w}{d r}=-\frac{W r}{4 \pi D}\left[\frac{\frac{1-v}{1+v}+\frac{b^{2}}{r^{2}}}{\frac{1-v}{1+v}+\frac{b^{2}}{a^{2}}}\right]\left[-\frac{1}{2}\left(1-\frac{d^{2}}{a^{2}}\right)+\ln \frac{a}{d}\right]
\end{aligned}
$$

$$
\begin{aligned}
& \text { Deflection }=\frac{W a^{2}}{8 \pi D}\left[\frac{1}{\frac{1-v}{1+v}+\frac{b^{2}}{a^{2}}}\right]\left\{\left(1-\frac{d^{2}}{a^{2}}\right)\left[\frac{1}{2}\left(\frac{1-v}{1+v}\right)\left(1+\frac{r^{2}}{a}\right)+\frac{b^{2}}{a^{2}}\right]-\left(1-\frac{d^{2}}{a^{2}}\right) \frac{b^{2}}{a^{2}} \ln \frac{d}{r}-\left[2 \frac{b^{2}}{a^{2}}+\left(\frac{1-v}{1+v}\right)\left(\frac{d^{2}}{a^{2}}+\frac{x^{2}}{a^{2}}\right)\right] \ln \frac{a}{d}+2 \frac{b^{2}}{a^{2}}\left(\ln \frac{a}{r}\right) \ln \frac{a}{d}\right\} \\
& w_{\max }=\frac{W^{2}}{8 \pi D}\left[\frac{1}{\frac{1-v}{1+v}+\frac{b^{2}}{a^{2}}}\right]\left\{\frac{1}{2}\left(1-\frac{d^{2}}{a^{2}}\right)\left[\frac{1-v}{1+v}+\left(\frac{3+v}{1+v}\right) \frac{b^{2}}{a^{2}}\right]-\left(1-\frac{a^{2}}{a^{2}}\right) \frac{b^{2}}{a^{2}} \ln \frac{d}{b}-\left[\left(\frac{1-v}{1+v}\right) \frac{d^{2}}{a^{2}}+\left(\frac{3+v}{1+v}\right) \frac{b^{2}}{a^{2}}\right] \ln \frac{a}{d}+2 \frac{b^{2}}{a^{2}}\left(\ln \frac{a}{b}\right) \ln \frac{a}{d}\right\}
\end{aligned}
$$

Outer portion of plate, $d \leq r \leq a$

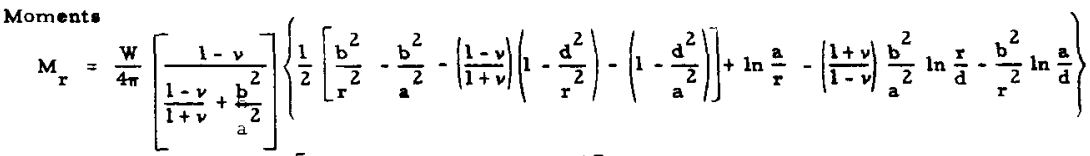

$$
\begin{aligned}
& M_{r a}=\frac{w}{2 \pi}\left[\frac{-1}{\frac{1-\nu}{1+\nu}+\frac{b^{2}}{a^{2}}}\right]\left[\frac{b^{2}}{a^{2}} \ln \frac{a}{d}+\frac{1}{2}\left(\frac{1-v}{1+v}\right)\left(1-\frac{d^{2}}{a^{2}}\right)\right] \\
& M_{t}=\frac{w}{4 r}\left[\frac{1-v}{\frac{1-v}{1+v}+\frac{b^{2}}{a^{2}}}\right]\left\{\frac{1}{2}\left[\left(\frac{1-v}{1+v}\right)\left(1-\frac{d^{2}}{r^{2}}\right)-\left(1-\frac{d^{2}}{a^{2}}\right)-\frac{b^{2}}{r^{2}}+\frac{b^{2}}{a^{2}}\right]+\ln \frac{a}{x}-\left(\frac{1+v}{1-v}\right) \frac{b^{2}}{a^{2}} \ln \frac{r}{d}+\frac{b^{2}}{r^{2}} \ln \frac{a}{d}\right\} \\
& M_{\text {ta }}=v M_{r a} \\
& \frac{d w}{d r}=\frac{W r}{4 \pi D}\left[\frac{-1}{\frac{1-v}{1+v}+\frac{b^{2}}{a^{2}}}\right]\left\{\frac{1}{2}\left[\frac{b^{2}}{a^{2}}-\frac{b^{2}}{r^{2}}-\left(\frac{1-v}{1+v}\right)\left(\frac{d^{2}}{x^{2}}-\frac{d^{2}}{a^{2}}\right)\right]+\left(\frac{1-v}{1+v}\right) \ln \frac{a}{r}-\frac{b^{2}}{a^{2}} \ln \frac{r}{d}+\frac{b^{2}}{r^{2}} \ln \frac{a}{d}\right\} \\
& w=\frac{W^{2}}{8 \pi D}\left[\frac{1}{\frac{1-v}{1+v}+\frac{b^{2}}{a^{2}}}\right]\left\{\left(1-\frac{r^{2}}{a^{2}}\right)\left[\frac{1}{2}\left(\frac{1-v}{1+v}\right)\left(1+\frac{d^{2}}{a^{2}}\right)+\frac{b^{2}}{a^{2}}\right]-\left[\frac{b^{2}}{2}+\left(\frac{l-v}{1+v}\right)\left(\frac{d^{2}}{a^{2}}+\frac{x^{2}}{a^{2}}\right)\right] \ln \frac{a}{x}+\frac{x^{2}}{a^{2}}\left(\frac{b^{2}}{a^{2}}\right) \ln \frac{x}{d}-\frac{b^{2}}{a^{2}} \ln \frac{a}{d}+2 \frac{b^{2}}{a^{2}}\left(\ln \frac{a}{r}\right) \ln \frac{a}{d}\right)
\end{aligned}
$$




\section{SIMPLIFIED CASES}

From the four generalized cases, six common, simplified cases were readily ascertained. The equations for the first four simplified cases were obtained by using the equations for the outer portion of the plate and letting $\mathrm{d}=\mathrm{b}$. Cases XI, XII, XIII, and XIV were derived from Cases VII, VIII, IX, and X, respectively. Using the equations for the inner portion of the plate and making $r=b=d$, enables the inner boundary equations to be checked. For Cases XV and XVI, the equations for the inner and outer portions were obtained by setting $b=0$. Case XV was derived from Case VII and then verified by Case X. Case XVI was formulated from Case VIII and checked by Case IX. 


\section{CASE XII}

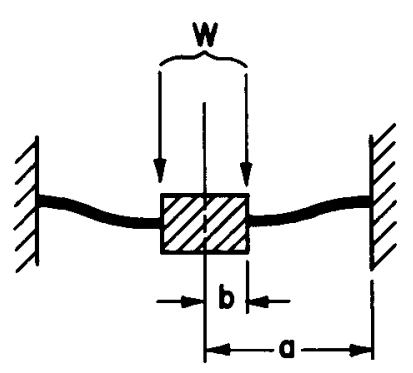

Description

Outer edge supported and fixed. Inner edge fixed.

Uniform load on inner concentric circle of plate.
Boundary Conditions

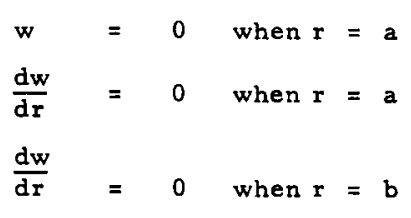

\section{Moments}

$$
\begin{aligned}
& M_{r}=\frac{w}{4 \pi}\left\{-1+(1+v)\left(\frac{a^{2}}{a^{2}-b^{2}}\right)\left[\ln \frac{a}{r}+\frac{b^{2}}{a^{2}} \ln \frac{r}{b}+\left(\frac{1-v}{1+v}\right) \frac{b^{2}}{r^{2}} \ln \frac{a}{b}\right]\right\} \\
& M_{r a}=\frac{w}{2 \pi}\left[-\frac{1}{2}+\frac{b^{2}}{a^{2}-b^{2}} \ln \frac{a}{b}\right] \\
& M_{\max }=M_{r b}=\frac{W}{2 \pi}\left[-\frac{1}{2}+\frac{a^{2}}{a^{2}-b^{2}} \ln \frac{a}{b}\right] \\
& M_{t}=\frac{W}{4 \pi}\left\{-v+(1+v)\left(\frac{a^{2}}{a^{2}-b^{2}}\right)\left[\ln \frac{a}{r}+\frac{b^{2}}{a^{2}} \ln \frac{r}{b}-\left\langle\frac{1-v}{1+v}\right| \frac{b^{2}}{r^{2}} \ln \frac{a}{b}\right\}\right\} \\
& M_{\text {ta }}=v M_{r a} ; \quad M_{t b}=v M_{r a}
\end{aligned}
$$

Slope

$$
\frac{d w}{d r}=-\frac{w r}{4 \pi D}\left(\frac{a^{2}}{a^{2}-b^{2}}\right)\left(\ln \frac{a}{r}+\frac{b^{2}}{a^{2}} \ln \frac{r}{b}-\frac{b^{2}}{r^{2}} \ln \frac{a}{b}\right)
$$

Deflection

$$
\begin{aligned}
& w=\frac{W a^{2}}{8 \pi D}\left(\frac{a^{2}}{a^{2}-b^{2}}\right)\left[\frac{1}{2}\left(1-\frac{r^{2}}{a^{2}}\right)\left(1-\frac{b^{2}}{a^{2}}\right)+\frac{b^{2}}{a^{2}}\left(1-\frac{r^{2}}{a^{2}}\right) \ln \frac{r}{b}+\left(\frac{b^{2}}{a^{2}}-\frac{r^{2}}{a^{2}}\right) \ln \frac{a}{r}-2 \frac{b^{2}}{a}\left(\ln \frac{a}{b}\right) \ln \frac{a}{r}\right] \\
& w_{\max }=\frac{W a^{2}}{8 \pi D}\left[\frac{l}{2}\left(1-\frac{b^{2}}{a^{2}}\right)-2 \frac{b^{2}}{a^{2}-b^{2}}\left(\ln \frac{a}{b}\right)^{2}\right]
\end{aligned}
$$


CASE XII

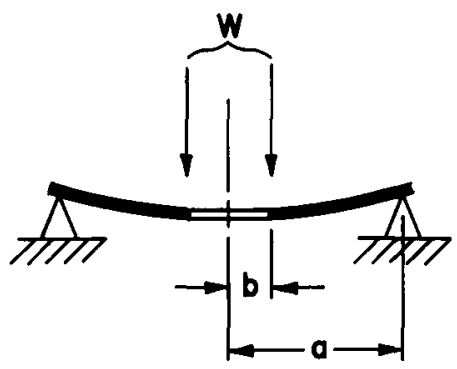

Description

Outer edge simply supported.

Inner edge free.

Uniform load on inner concentric circle of plate.
Boundary Conditions

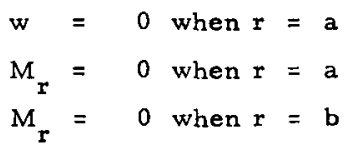

Moments

$$
\begin{aligned}
& M_{r}=\frac{W}{4 \pi}(1+v)\left(\frac{a^{2}}{a^{2}-b^{2}}\right)\left[\ln \frac{a}{r}+\frac{b^{2}}{a^{2}} \ln \frac{r}{b}-\frac{b^{2}}{r^{2}} \ln \frac{a}{b}\right] \\
& M_{t}=\frac{w}{4 \pi}(1+v)\left(\frac{a^{2}}{a^{2}-b^{2}}\right)\left[\left(\frac{1-v}{1+v}\right)\left(1-\frac{b^{2}}{a^{2}}\right)+\ln \frac{a}{r}+\frac{b^{2}}{a^{2}} \ln \frac{r}{b}+\frac{b^{2}}{r^{2}} \ln \frac{a}{b}\right] \\
& M_{t a}=\frac{W}{2 \pi}(1+v)\left[\frac{l}{2}\left(\frac{1-v}{I+v}\right)+\frac{b^{2}}{a^{2}-b^{2}} \ln \frac{a}{b}\right] \\
& M_{\max }=M_{t b}=\frac{W}{2 \pi}(1+v)\left[\frac{1}{2}\left(\frac{1-v}{1+v}\right)+\frac{a^{2}}{a^{2}-b^{2}} \ln \frac{a}{b}\right]
\end{aligned}
$$

Slope

$$
\frac{d w}{d r}=-\frac{w r}{4 \pi D}\left(\frac{a^{2}}{a^{2}-b^{2}}\right)\left[\left(\frac{1}{1+v}\right)\left(1-\frac{b^{2}}{a^{2}}\right)+\ln \frac{a}{r}+\frac{b^{2}}{a^{2}} \ln \frac{r}{b}+\left(\frac{1+v}{1-v}\right) \frac{b^{2}}{r^{2}} \ln \frac{a}{b}\right]
$$

Deflection

$$
\begin{aligned}
& w=\frac{w a^{2}}{8 \pi D}\left[\frac{1}{2}\left(\frac{3+v}{1+v}\right)\left(1-\frac{r^{2}}{a^{2}}\right)-\frac{r^{2}}{a^{2}-b^{2}} \ln \frac{a}{r}+\frac{b^{2}}{a^{2}-b^{2}}\left(\ln \frac{a}{b}-\frac{r^{2}}{a^{2}} \ln \frac{r}{b}\right)+2\left(\frac{1+v}{1-v}\right)\left(\frac{b^{2}}{a^{2}-b^{2}}\right)\left(\ln \frac{a}{r}\right)\left(\ln \frac{a}{b}\right)\right] \\
& w_{\max }=\frac{w_{a}^{2}}{8 \pi D}\left[\frac{1}{2}\left(\frac{3+v}{1+v}\right)\left(1-\frac{b^{2}}{a^{2}}\right)+2\left(\frac{1+v}{1-v}\right)\left(\frac{b^{2}}{a^{2}-b^{2}}\right)\left(\ln \frac{a}{b}\right)^{2}\right]
\end{aligned}
$$


CASE XIII

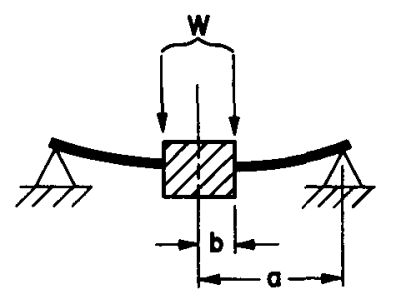

Description

Outer edge simply supported. Inner edge fixed.

Uniform load on inner concentric circle of plate.
Boundary Conditions

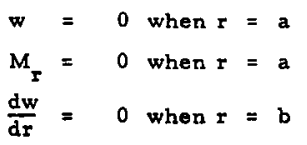

Moments

$$
\begin{aligned}
& M_{r}=\frac{W}{4 \pi}\left[\frac{1+v}{\frac{1+v}{1-v}+\frac{b^{2}}{a^{2}}}\right]\left[\left(\frac{1}{1+v}\right)\left(1-\frac{r^{2}}{a^{2}}\right) \frac{b^{2}}{r^{2}}+\frac{b^{2}}{r^{2}} \ln \frac{a}{b}+\left(\frac{1+v}{1-v}\right) \ln \frac{a}{r}-\frac{b^{2}}{a^{2}} \ln \frac{r}{b}\right] \\
& M_{\max }=M_{r b}=\frac{W}{2 \pi}\left[\frac{1}{\frac{1+v}{1-v}+\frac{b^{2}}{a^{2}}}\right]\left[\frac{1}{2}\left(1-\frac{b^{2}}{a^{2}}\right)+\left(\frac{1+v}{1-v}\right) \ln \frac{a}{b}\right] \\
& M_{t}=\frac{W}{4 \pi}\left[\frac{1+v}{1+v}\right]\left[1-\left(\frac{b^{2}}{1+v}\right) \frac{b^{2}}{a^{2}}-\left(\frac{1}{1+v}\right) \frac{b^{2}}{a^{2}}+\left(\frac{1+v}{1-v}\right) \ln \frac{a}{r}-\frac{b^{2}}{r^{2}} \ln \frac{a}{b}-\frac{b^{2}}{a} \ln \frac{r}{b}\right] \\
& M_{t a}=\frac{W}{2 \pi}\left[\frac{1+v}{1+v}\right]\left[\frac{1}{2}\left(1-\frac{b^{2}}{a^{2}}\right)-\frac{b^{2}}{a^{2}} \ln \frac{a}{b}\right] \\
& M_{t b}=v M_{r b}
\end{aligned}
$$

slope

$$
\frac{d w}{d r}=-\frac{W r}{4 \pi D}\left[\frac{1}{\frac{1+v}{1-v}+\frac{b^{2}}{a^{2}}}\right]\left[\left(\frac{1}{1-v} \mid\left(1-\frac{b^{2}}{r^{2}}\right)+\left(\frac{1+v}{1-v}\right) \ln \frac{a}{r}-\left(\frac{1+v}{1-v}\right) \frac{b^{2}}{x^{2}} \ln \frac{a}{b}-\frac{b^{2}}{a^{2}} \ln \frac{r}{b}\right]\right.
$$

Deflection

$$
\begin{aligned}
& w=\frac{w^{2}}{8 \pi D}\left[\frac{1}{\frac{1+v}{1-v}+\frac{b^{2}}{a^{2}}}\right]\left\{\frac{1}{2}\left(1-\frac{r^{2}}{a^{2}}\right)\left(\frac{3+v}{1-v}+\frac{b^{2}}{a^{2}}\right)-\left(\frac{1+v}{1-v}\right)\left[\left(\frac{2}{1+v}\right) \frac{b^{2}}{a^{2}}+\frac{r^{2}}{a^{2}}\right] \ln \frac{a}{r}-\frac{b^{2}}{a^{2}} \ln \frac{a}{b}+\frac{r^{2}}{a^{2}}\left(\frac{b^{2}}{a^{2}}\right) \ln \frac{r}{b}-2\left(\frac{1+v}{1-v}\right)\left|\frac{b^{2}}{a}\right|\left(\ln \frac{a}{r}\right) \ln \frac{a}{b}\right\} \\
& \left.\left.w_{\max }=\frac{w a^{2}}{8 \pi D}\left[\frac{1}{\frac{1+v}{1-v}+\frac{b^{2}}{a^{2}}}\right]^{-\frac{1}{2}}\left(1-\frac{b^{2}}{a^{2}}\right)\left(\frac{3+v}{1-v}+\frac{b^{2}}{a^{2}}\right)-\left(\frac{4}{1-v}\right) \frac{b^{2}}{a^{2}} \ln \frac{a}{b}-2\left(\frac{1+v}{1-v}\right)\left(\frac{b^{2}}{a^{2}}\right) \mid \ln \frac{a}{b}\right)^{2}\right]
\end{aligned}
$$




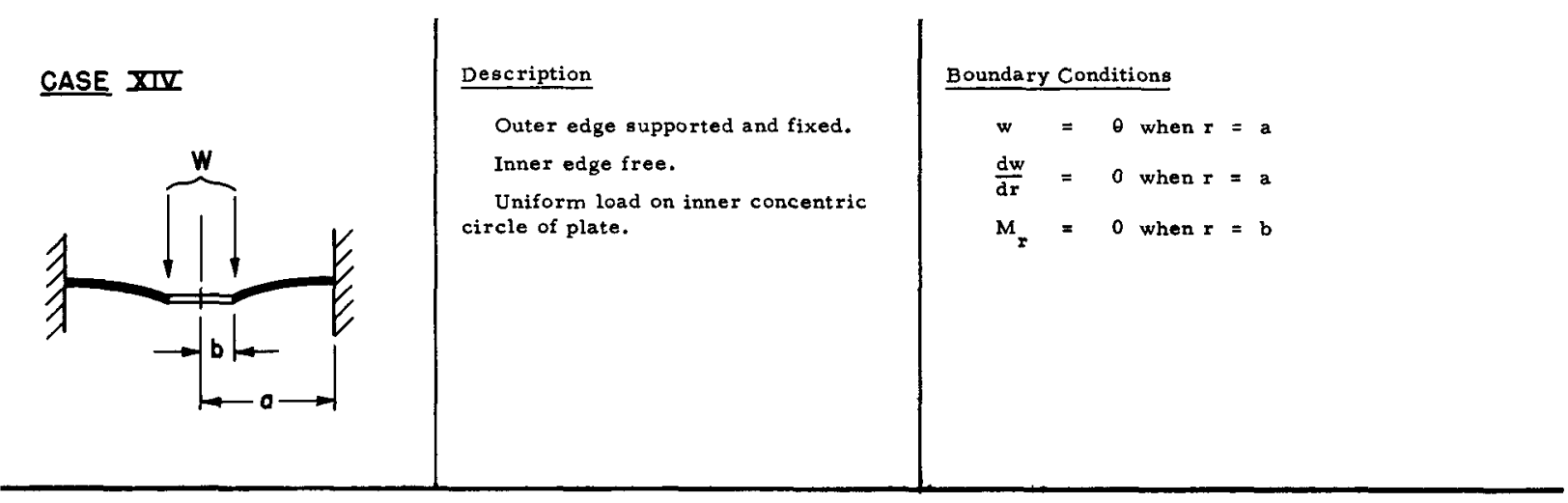

Moments

$$
\begin{aligned}
& M_{r}=\frac{w}{4 \pi}\left[\frac{1-v}{\frac{1-v}{1+v}+\frac{b^{2}}{a^{2}}}\right]\left[\left(\frac{-1}{1+v}\right)\left(1-\frac{b^{2}}{x^{2}}\right)+\ln \frac{a}{x}-\left(\frac{1+v}{1-v}\right) \frac{b^{2}}{a^{2}} \ln \frac{r}{b}-\frac{b^{2}}{r^{2}} \ln \frac{a}{b}\right] \\
& M_{r a}=\frac{w}{2 \pi}\left[\frac{-1}{\frac{1-v}{1+v}+\frac{b^{2}}{a^{2}}}\right]\left[\frac{1}{2}\left(\frac{1-v}{1+v}\right)\left(1-\frac{b^{2}}{a^{2}}\right)+\frac{b^{2}}{a^{2}} \ln \frac{a}{b}\right] \quad \text { (Maximum when } a / b<2.6 ; \quad v=0.3 \text { ) } \\
& M_{t}=\frac{w}{4 \pi}\left[\frac{1-y}{\frac{1-v}{1+\nu}+\frac{b^{2}}{a^{2}}}\right]\left[-\frac{v}{1+v}-\left(\frac{1}{1+v}\right) \frac{b^{2}}{r^{2}}+\frac{b^{2}}{a^{2}}+\ln \frac{a}{x}-\left(\frac{1+v}{1-v}\right) \frac{b^{2}}{a^{2}} \ln \frac{r}{b}+\frac{b^{2}}{x^{2}} \ln \frac{a}{b}\right] \\
& M_{t b}=\frac{w}{2 \pi}\left[\frac{-(1-v)}{\frac{1-\nu}{1+\nu}+\frac{b^{2}}{a^{2}}}\right]\left[\frac{1}{2}\left(1-\frac{b^{2}}{a^{2}}\right)-\ln \frac{a}{b}\right] \quad(\text { Maximurn when } a / b>2.6 ; v=0.3)
\end{aligned}
$$

$M_{t a}=\nu M_{r a}$

Slope

$$
\frac{d w}{d r}=\frac{w r}{4 \pi D}\left[\frac{-1}{\frac{1-\nu}{1+\nu}+\frac{b^{2}}{a}}\right]\left[\left(\frac{1}{1+\nu}\right)\left(\frac{b^{2}}{a^{2}}-\frac{b^{2}}{r^{2}}\right)+\left(\frac{1-v}{1+\nu}\right) \ln \frac{a}{r}-\frac{b^{2}}{a^{2}} \ln \frac{r}{b}+\frac{b^{2}}{r^{2}} \ln \frac{a}{b}\right]
$$

Deflection

$$
\begin{aligned}
& \left.w=\frac{W a}{8 \pi D}\left[\frac{1}{\frac{1-v}{1+v}+\frac{b^{2}}{a}}\right]\left\{\frac{1}{2}\left(1-\frac{x^{2}}{a^{2}}\right)\left[\frac{1-v}{1+v}+\left(\frac{3+v}{1+v}\right) \frac{b^{2}}{a^{2}}\right]-\left[\left(\frac{2}{1+v}\right) \frac{b^{2}}{a^{2}}+\left(\frac{1-v}{1+v}\right) \frac{x^{2}}{a^{2}}\right] \ln \frac{a}{x}+\frac{x^{2}}{a} \mid \frac{b^{2}}{a^{2}}\right) \ln \frac{x}{b}+\frac{b^{2}}{a^{2}} \ln \frac{a}{b}+2 \frac{b^{2}}{a}\left|\ln \frac{a}{x}\right| \ln \frac{a}{b}\right\} \\
& w_{\max }=\frac{w_{a}^{2}}{8 \pi D}\left[\frac{1}{\frac{1-v}{1+v}+\frac{b^{2}}{a^{2}}}\right]\left\{\frac{1}{2}\left(1-\frac{b^{2}}{a^{2}}\right) \frac{1-v}{1+v}+\left(\frac{3+v}{1+v}\right) \frac{b^{2}}{a^{2}}\right]-\left(\left.\frac{4}{1+v}\left|\frac{b^{2}}{a^{2}} \ln \frac{a}{b}+2 \frac{b^{2}}{a^{2}}\right| \ln \frac{a}{b}\right|^{2}\right\}
\end{aligned}
$$




\begin{tabular}{|c|c|c|}
\hline CASE XV & Description & Boundary Conditions \\
\hline$\frac{\lambda}{\lambda}$ & $\begin{array}{l}\text { Outer edge supported and fixed. } \\
\text { Solid Plate. } \\
\text { Uniform load on a concentric } \\
\text { circle of plate. }\end{array}$ & 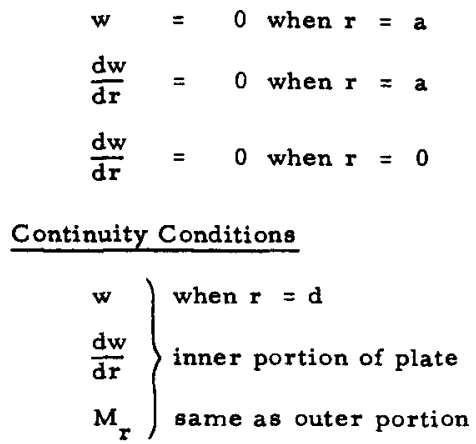 \\
\hline
\end{tabular}

Inner portion of plate $0 \leq r \leq d$

Moments

$$
M_{r}=M_{t}=M_{r d}=M_{t d}=\frac{W}{4 \pi}(1+v)\left[\ln \frac{a}{d}-\frac{1}{2}\left(1-\frac{d^{2}}{a^{2}}\right)\right] \quad(\text { Maximum when } a / d>3.13 ; v=0.3)
$$

Slope

$$
\frac{d w}{d \mathbf{r}}=-\frac{w r}{4 \pi D}\left[\ln \frac{a}{d}-\frac{1}{2}\left(1-\frac{d^{2}}{a^{2}}\right)\right]
$$

Doflection

$$
\begin{aligned}
& w=\frac{w a^{2}}{8 \pi D}\left[\frac{1}{2}\left(1-\frac{d^{2}}{a^{2}}\right)\left(1+\frac{r^{2}}{a^{2}}\right)-\left(\frac{d^{2}}{a^{2}}+\frac{r^{2}}{a^{2}}\right) \ln \frac{a}{d}\right] \\
& w_{\max }=\frac{w a^{2}}{8 \pi D}\left[\frac{1}{2}\left(1-\frac{d^{2}}{a^{2}}\right)-\frac{d^{2}}{a^{2}} \ln \frac{a}{d}\right]
\end{aligned}
$$

Outer portion of plate

Moments

$$
\begin{aligned}
& M_{r}=\frac{W}{4 \pi}(1+v)\left\{\ln \frac{a}{r}-\frac{1}{2}\left[\left(\frac{1-v}{1+v}\right)\left(1-\frac{d^{2}}{z^{2}}\right)+\left(1-\frac{d^{2}}{a^{2}}\right)\right]\right\} \\
& \left.M_{r a}=-\frac{W}{4 \pi}\left(1-\frac{d^{2}}{a^{2}}\right) \quad \text { (Maximum when } a / d<3.13 ; v=0.3\right) \\
& M_{t}=\frac{W}{4 \pi}(1+v)\left\{\ln \frac{a}{r}+\frac{1}{2}\left[\left(\frac{1-v}{1+v}\right)\left(1-\frac{d^{2}}{r^{2}}\right)-\left(1-\frac{d^{2}}{a^{2}}\right)\right]\right\} \\
& M_{t a}=v M_{r a}
\end{aligned}
$$

Slope

$$
\frac{d w}{d r}=-\frac{w r}{4 \pi D}\left[\ln \frac{a}{r}-\frac{1}{2}\left(\frac{d^{2}}{r^{2}}-\frac{d^{2}}{a^{2}}\right)\right]
$$

Deflection

$$
w=\frac{W a^{2}}{8 \pi D}\left[\frac{1}{2}\left(1+\frac{d^{2}}{a^{2}}\right)\left(1-\frac{r^{2}}{a^{2}}\right)-\left(\frac{d^{2}}{a^{2}}+\frac{r^{2}}{a^{2}}\right) \ln \frac{a}{r}\right]
$$


CASE XVI

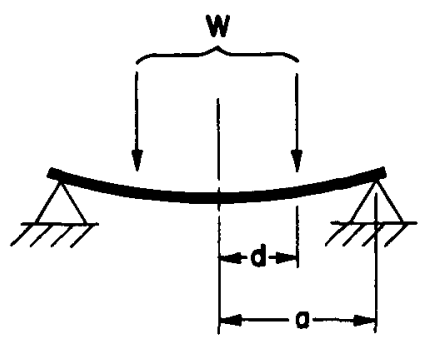

Description

Outer edge simply supported.

Solid plate.

Uniform load on a concentric circle of plate.
Boundary Conditions

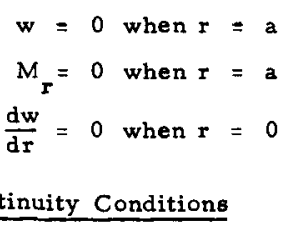

w when $r=d$ $\frac{d w}{d r}$ inner portion of plate $\left.M_{r}\right\}$ same as outer portion

Inner portion of plate $0 \leq \mathrm{r} \leq \mathrm{d}$

Momente

$$
M_{\max }=M_{r}=M_{t}=M_{r d}=M_{t d}=\frac{W}{4 \pi}(1+\nu)\left[\frac{1}{2}\left(\frac{1-v}{1+\nu}\right)\left(1-\frac{d^{2}}{a^{2}}\right)+\ln \frac{a}{d}\right]
$$

Slope

$$
\frac{d w}{d r}=-\frac{W r}{4 \pi D}\left[\frac{1}{2}\left(\frac{1-v}{1+v}\right)\left(1-\frac{d^{2}}{a^{2}}\right)+\ln \frac{a}{d}\right]
$$

$$
\begin{aligned}
& \text { Deflection } \\
& w=\frac{W a^{2}}{8 \pi D}\left\{\frac{1}{2}\left(1-\frac{d^{2}}{a^{2}}\right)\left[\frac{3+v}{1+v}-\left(\frac{1-v}{1+v}\right) \frac{r^{2}}{a^{2}}\right]-\left(\frac{r^{2}}{a^{2}}+\frac{d^{2}}{a^{2}}\right) \ln \frac{a}{d}\right\} \\
& w_{\max }=\frac{W a^{2}}{8 \pi D}\left[\frac{1}{2}\left(\frac{3+v}{1+v}\right)\left(1-\frac{d^{2}}{a^{2}}\right)-\frac{d^{2}}{a^{2}} \ln \frac{a}{d}\right]
\end{aligned}
$$

Outer portion of plate $d \leq r \leq a$

Moments

$$
\begin{aligned}
& M_{r}=\frac{W}{4 \pi}(1+v)\left[\frac{1}{2}\left(\frac{1-v}{1+v}\right)\left(\frac{d^{2}}{r^{2}}-\frac{d^{2}}{a^{2}}\right)+\ln \frac{a}{r}\right] \\
& M_{t}=\frac{W}{4 \pi}(1+v)\left[\frac{1}{2}\left(\frac{1-v}{1+v}\right)\left(2-\frac{d^{2}}{r^{2}}-\frac{d^{2}}{a^{2}}\right)+\ln \frac{a}{r}\right] \\
& M_{t a}=\frac{W}{4 \pi}(1-v)\left(1-\frac{d^{2}}{a^{2}}\right)
\end{aligned}
$$

Slope

$$
\frac{d w}{d r}=-\frac{W r}{4 \pi D}\left\{\frac{1}{2}\left[\left(1-\frac{d^{2}}{r^{2}}\right)+\left(\frac{1-v}{1+v}\right)\left(1-\frac{d^{2}}{a^{2}}\right)\right]+\ln \frac{a}{r}\right\}
$$

Deflection

$$
w=\frac{W a^{2}}{8 \pi D}\left\{\frac{1}{2}\left(1-\frac{r^{2}}{a^{2}}\right)\left[\frac{3+v}{1+v}-\left(\frac{1-v}{1+v}\right) \frac{d^{2}}{a^{2}}\right]-\left(\frac{d^{2}}{a^{2}}+\frac{r^{2}}{a^{2}}\right) \ln \frac{a}{r}\right\}
$$




\section{DESIGN CONSIDERATIONS}

Normally, either the maximum bending moment or the maximum deflection establishes the design criteria for thin, flat, circular plates. The maximum deflection can be expressed by a formula of the type

$$
\mathrm{w}_{\max }=\mathrm{Wa}^{2} / \mathrm{Eh}^{3} .
$$

The maximum bending moment can be represented by the expression

$$
\mathrm{M}_{\max }=\mathrm{k}_{\mathrm{m}} \mathrm{W},
$$

where "maximum" denotes magnitude only, or maximum absolute value.

Since the absolute maximum bending moment determines the maximum unit stresses, its location and magnitude must be known. Because of the complexity of the moment equations for the four generalized cases and the dependency of Poisson's ratio upon the material and other parameters, only the absolute maximum bending moment of Case VIII could be established by studying the derived equations for any combinations of $a / b$ and $d / b$ or $\mathrm{a} / \mathrm{d}$ ratios and Poisson's ratio. The maximum bending moments for Cases XI, XII, XIII, and XVI, the four simplified cases having any Poisson's ratio and $\mathrm{a} / \mathrm{b}$ or $\mathrm{a} / \mathrm{d}$ ratio, were resolved by examining the equations. Theoretically, Poisson's ratio has a value from zero to one-half; e.g., for materials like paraffin and rubber, the ratios are almost one-half; for cork, the ratio is approximately zero. Poisson's ratio was taken to be 0.3 , as is normally done, and the maximum bending moment and its location were determined for simplified Cases XIV and XV.

Table I contains various terms in the derived moment, slope, and deflection formulas to facilitate computations.

To eliminate numerous laborious hand computations, a computer program was developed. This Argonne National Laboratory program $2117 /$ PADl 46 computes and tabulates the deflection constants, the radial moment per load, and the tangential moment per load at predetermined $\mathrm{r} / \mathrm{b}$ or $\mathrm{r} / \mathrm{d}$ ratios, and for any given combination of values for $\nu$ and ratios $\mathrm{a} / \mathrm{b}$ and $\mathrm{d} / \mathrm{b}$ or $\mathrm{a} / \mathrm{d}$. For Cases VII through XIV, the tabulated values of $\mathrm{r} / \mathrm{b}$ range from one to the selected value of $\mathrm{a} / \mathrm{b}$, in increments of 0.1 . Also, Cases VII through X include $r / b=d / b$. For Cases XV and XVI, r $/ d$ varies from zero to the selected value of $a / d$, also in increments of 0.1 .

To acquire a better insight into the bending moments of these ten cases, especially the four generalized cases, numerical data for the radial and tangential moments divided by the load, plus the deflection constant, were ascertained with the aforementioned program. The following data 
TABLE

COMPUTATION TERMS

\begin{tabular}{|c|c|c|c|c|c|c|c|c|c|c|c|c|c|c|c|c|}
\hline$\frac{a}{b}$ & $\frac{b^{2}}{a^{2}}$ & $1-\frac{b^{2}}{a^{2}}$ & $1+\frac{b^{2}}{a^{2}}$ & $\frac{b^{2}}{a^{2}-b^{2}}$ & $\frac{a^{2}}{a^{2}-b^{2}}$ & $\ln \frac{a}{b}$ & $\left|\ln \frac{a}{b}\right|^{2}$ & $\ln \frac{a}{b}-1$ & $\mid\left(\ln \frac{a}{b}-\left.1\right|^{2}\right.$ & $\frac{b^{2}}{a^{2}}\left\{\ln \frac{a}{b}\right\}$ & $\left.\frac{b^{2}}{a^{2}} \mid \ln \frac{a}{b}\right)^{2}$ & $\left(1+\frac{b^{2}}{a^{2}}\right) \ln \frac{a}{b}$ & $\frac{b^{2}}{a^{2}-b^{2}}\left|\ln \frac{a}{b}\right|$ & $\frac{b^{2}}{a^{2}-b^{2}}\left|\ln \frac{a}{b}\right|^{2}$ & $\frac{a^{2}}{a^{2}-b^{2}}\left|\ln \frac{a}{b}\right|$ & $\frac{a^{2}}{a^{2}-b^{2}}\left|\ln \frac{a}{b}\right|^{2}$ \\
\hline 1.0 & 1.00000 & 0.00000 & 2.00000 & $\infty$ & $\infty$ & 0.00000 & 0.00000 & -1.00000 & 1.00000 & 0.00000 & 0.00000 & 0.00000 & 0.50000 & 0.00000 & 0.50000 & 0.00000 \\
\hline 1,1 & 0.82645 & 0.17355 & 1.82645 & 4.76190 & 5.76190 & 0.09531 & 0.00908 & -0.90469 & 0.81846 & 0.07877 & 0.00750 & 0.17408 & 0.45386 & 0.04324 & 0.54917 & 0.05232 \\
\hline 1.2 & 0.69444 & 0.30556 & 1.69444 & 2.27273 & 3.27273 & 0.18232 & 0.03324 & -0.81768 & 0.66860 & 0.12661 & 0.02308 & 0.30893 & 0.41436 & 0.07555 & 0.59668 & 0.10879 \\
\hline 1.3 & 0.59172 & 0.40828 & 1.59172 & 1.44928 & 2.44928 & 0.26236 & 0.06883 & -0.73764 & 0.54411 & 0.15524 & 0.04073 & 0.41760 & 0.38023 & 0.09975 & 0.64259 & 0.16858 \\
\hline 1.4 & 0.51020 & 0.48980 & 1.51020 & 1.04167 & 2.04167 & 0.33647 & 0.11321 & -0.66353 & 0.44027 & 0.17167 & 0.05776 & 0.50814 & 0.35049 & 0.11793 & 0.68696 & 0.23114 \\
\hline 1.5 & 0.44444 & 0.55556 & 1.44444 & 0.80000 & 1.80000 & 0.40547 & 0.16441 & -0.59453 & 0.35347 & 0.18021 & 0.07307 & 0.58568 & 0.32438 & 0.13153 & 0.72985 & 0.29594 \\
\hline 1.6 & 0.39063 & 0.60937 & 1.39063 & 0.64103 & 1.64103 & 0.47000 & 0.22090 & -0.53000 & 0.28090 & 0.18360 & 0.08629 & 0.65360 & 0.30128 & 0.14160 & 0.77128 & 0.36250 \\
\hline 1.7 & 0.34602 & 0.65398 & 1.34602 & 0.52910 & 1.52910 & 0.53063 & 0.28157 & -0.46937 & 0.22031 & 0.18361 & 0.09743 & 0.71424 & 0.28076 & 0.14898 & 0.81139 & 0.43055 \\
\hline 1.8 & 0.30864 & 0.69136 & 1.30864 & 0.44643 & 1.44643 & 0.58779 & 0.34550 & -0.41221 & 0.16992 & 0.18142 & 0.10664 & 0.76921 & 0.26241 & 0.15424 & 0.85020 & 0.49974 \\
\hline 1.9 & 0.27701 & 0.72299 & 1.27701 & 0.38314 & 1.38314 & 0.64185 & 0.41197 & -0.35815 & 0.12827 & 0.17780 & 0.11412 & 0.81965 & 0.24592 & 0.15784 & 0.88777 & 0.56981 \\
\hline 2.0 & 0.25000 & 0.75000 & 1.25000 & 0.33333 & 1.33333 & 0.69315 & 0.48046 & -0.30685 & 0.09416 & 0.17329 & 0.12012 & 0.86644 & 0.23105 & 0.16015 & 0.92420 & 0.64061 \\
\hline 2.1 & 0.22676 & 0.77324 & 1.22676 & 0.29326 & 1.29326 & 0.74194 & 0.55047 & -0.25806 & 0.06659 & 0.16824 & 0.12482 & 0.91018 & 0.21758 & 0.16143 & 0.95952 & 0.71190 \\
\hline 2.2 & 0.20661 & 0.79339 & 1.20661 & 0.26042 & 1.26042 & 0.78846 & 0,62167 & -0.21154 & 0.04475 & 0.16290 & 0.12844 & 0.95136 & 0.20533 & 0.16189 & 0.99379 & 0.78357 \\
\hline 2.3 & 0.18904 & 0.81096 & 1.18904 & 0.23310 & 1.23310 & 0.83291 & 0.69374 & -0.16709 & 0.02792 & 0.15745 & 0.13114 & 0.99036 & 0.19415 & 0.16171 & 1.02706 & 0.85545 \\
\hline 2.4 & 0.17361 & 0.82639 & 1.17361 & 0.21008 & 1.21008 & 0.87547 & 0.76645 & -0.12453 & 0.01551 & 0.15199 & 0.13306 & 1.02746 & 0.18392 & 0.16102 & 1.05939 & 0.92747 \\
\hline 2.5 & 0.16000 & 0.84000 & 1.16000 & 0.19048 & 1.19048 & 0.91629 & 0.83959 & -0.08371 & 0.00701 & 0.14661 & 0.13433 & 1.06290 & 0.17453 & 0.15992 & 1.09082 & 0.99952 \\
\hline 2.6 & 0.14793 & 0.85207 & 1.14793 & 0.17361 & 1.17361 & 0.95551 & 0.91300 & -0.04449 & 0.00198 & 0.14135 & 0.13506 & 1.09686 & 0.16589 & 0.15851 & 1.12140 & 1.07151 \\
\hline 2.7 & 0.13717 & 0.86283 & 1.13717 & 0.15898 & 1.15898 & 0.99325 & 0.98655 & -0.00675 & 0.00005 & 0.13624 & 0.13533 & 1. 12949 & 0.15791 & 0.15684 & 1.15116 & 1.14339 \\
\hline 2.8 & 0.12755 & 0.87245 & 1.12755 & 0.14620 & 1.14620 & 1.02962 & 1.06012 & 0.02962 & 0.00088 & 0.13133 & 0.13522 & 1,16095 & 0.15053 & 0.15499 & 1.18015 & 1.21511 \\
\hline 2.9 & 0.11891 & 0.88109 & 1.11891 & 0.13495 & 1.13495 & 1.06471 & 1.13361 & 0.06471 & 0.00419 & 0.12660 & 0.13480 & 1.19131 & 0.14369 & 0.15298 & 1.20839 & 1.28659 \\
\hline 3.0 & 0.11111 & 0.88889 & 1.11111 & 0.12500 & 1.12500 & 1.09861 & 1.20694 & 0.09861 & 0.00972 & 0.12207 & 0.13410 & 1.22068 & 0.13733 & 0.15087 & 1.23594 & 1.35781 \\
\hline 3.1 & 0.10406 & 0.89594 & 1.10406 & 0.11614 & 1.11614 & 1.13140 & 1.28007 & 0.13140 & 0.01727 & 0.11773 & 0.13320 & 1.24913 & 0.13141 & 0.14867 & 1.26280 & 1.42874 \\
\hline 3.2 & 0.09766 & 0.90234 & 1.09766 & 0.10823 & 1.10823 & 1.16315 & 1.35292 & 0.16315 & 0.02662 & 0.11359 & 0.13213 & 1.27674 & 0.12588 & 0.14642 & 1.28904 & 1.49935 \\
\hline 3.3 & 0.09183 & 0.90817 & 1.09183 & 0.10111 & 1.10111 & 1,19392 & 1.42544 & 0.19392 & 0.03760 & 0.10964 & 0.13090 & 1.30356 & 0.12072 & 0.14413 & 1.31464 & 1.56957 \\
\hline 3.4 & 0.08651 & 0.91349 & 1.08651 & 0.09470 & 1.09470 & 1.22378 & 1.49764 & 0.22378 & 0.05008 & 0.10587 & 0.12956 & 1.32965 & 0.11589 & 0.14182 & 1.33967 & 1.63947 \\
\hline 3.5 & 0.08163 & 0.91837 & 1.08163 & 0.08889 & 1.08889 & 1.25276 & 1.56941 & 0.25276 & 0.06389 & 0.10226 & 0.12811 & 1.35502 & 0.11136 & 0.13950 & 1.36412 & 1.70891 \\
\hline 3.6 & 0.07716 & 0.92284 & 1.07716 & 0.08361 & 1.08361 & 1.28093 & 1.64078 & 0.28093 & 0.07892 & 0.09884 & 0.12660 & 1.37977 & 0.10710 & 0.13719 & 1.38803 & 1.77797 \\
\hline 3.7 & 0.07305 & 0.92695 & 1.07305 & 0.07880 & 1.07880 & 1.30833 & 1.71173 & 0.30833 & 0.09507 & 0.09557 & 0.12300 & 1.40390 & 0.10310 & 0.13489 & 1.41143 & 1.84661 \\
\hline 3.8 & 0.06925 & 0.93075 & 1.06925 & 0.07440 & 1.07440 & 1.33500 & 1.78223 & 0.33500 & 0.11223 & 0.09245 & 0.12342 & 1.42745 & 0.09933 & 0.13261 & 1.43432 & 1.91483 \\
\hline 3.9 & 0.06575 & 0.93425 & 1.06575 & 0.07037 & 1.07037 & 1.36098 & 1.85227 & 0.36098 & 0.13031 & 0.08948 & 0.12179 & 1.45046 & 0.09578 & 0.13035 & 1.45675 & 1.98261 \\
\hline 4.0 & 0.06250 & 0.93750 & 1.06250 & 0.06667 & 1.06667 & 1.38629 & 1.92180 & 0.38629 & 0.14922 & 0.08664 & 0.12011 & 1.47293 & 0.09242 & 0.12812 & 1.47871 & 2.04993 \\
\hline
\end{tabular}


were used: (1) Poisson's ratio equals 0.3 for all cases; (2) a/b for Cases VII through XIV, and a/d for Cases XV and XVI, range from 1.5 through 4.0, in intervals of 0.5 ; and (3) the uniform load on a concentric circle for the four generalized cases is equally positioned at two locations between the inner and outer plate radii i.e., the third distance of $d=b+(a-b) / 3$ or $d / b=$ $(a / b+2) / 3$, and $d=b+(2 / 3)(a-b)$ or $d / b=(2 a / b+1) / 3$.

Numerical values of the maximum deflection constant and maximum bending moment per load are tabulated in Table II using the stipulations of the preceding paragraph. Figures 2, 3, 4, and 5 depict the bending moments per load for the generalized cases for $a / b$ ratios of $2.0,3.0$, and 4.0 . The bending-moments-per-load diagrams for the six simplified cases are illustrated by Figs. 6 through 11 .

The following statements can be made for the maximum bending moment and its location, with the aforementioned prescribed criteria: studied.

Case VII. Mra has the maximum bending moment for all ratios Case VIII.
studied. Case IX. Mrb has the maximum bending moment for all ratios studied. studied.

Case X. Mra has the maximum bending moment for all ratios

Case XI. $M_{r b}$ has the maximum bending moment for all ratios studied, as predicted from equation study.

Case XII. $M_{t b}$ has the maximum bending moment for all ratios studied, as predicted from equation study.

Case XIII. Mrb has the maximum bending moment for all ratios studied, as predicted from equation study.

Case XIV. The maximum bending moment must be established according to specifications. $M_{r a}$ has the maximum bending moment from $\mathrm{a} / \mathrm{b}=1.5$ through 2.5 , and then $\mathrm{M}_{\mathrm{tb}}$ has the maximum bending moment from 3.0 through 4.0 . The transition $\mathrm{a} / \mathrm{b}$ ratio is approximately 2.6 .

Case XV. The maximum bending moment must be determined according to specifications. $M_{x}$ a has the maximum bending moment from 1.5 through 3.0, and then the constant moments of the inner portion of the plate $\left(M_{r}=M_{t}=M_{r d}=M_{t d}\right)$ predominate from 3.5 through 4.0. The transition $\mathrm{a} / \mathrm{b}$ ratio is approximately 3.13 .

CaseXVI. Moments of the inner portion of the plate were maximum, constant, and equivalent for all ratios as predicted from equation study. 
TABLE II

MAXIMUM DEFLECTION AND MOMENT CONSTANTS WHERE $v=0.3$

\begin{tabular}{|c|c|c|c|c|c|c|c|c|c|c|}
\hline \multirow{2}{*}{$a / b$} & \multirow{2}{*}{$\begin{array}{l}\frac{\mathrm{d}}{\mathrm{b}}=\frac{\mathrm{a} / \mathrm{b}+2}{3} \\
\frac{\mathrm{d}}{\mathrm{b}}=\frac{2 \mathrm{a} / \mathrm{b}+1}{3}\end{array}$} & \multirow{2}{*}{$\mathrm{a} / \mathrm{d}$} & \multicolumn{2}{|c|}{ Case VII } & \multicolumn{2}{|c|}{ Case VIII } & \multicolumn{2}{|c|}{ Case IX } & \multicolumn{2}{|c|}{ Case X } \\
\hline & & & $\mathrm{k}_{\mathrm{d}}$ & $\mathrm{k}_{\mathrm{m}}$ & $k_{d}$ & $\mathrm{k}_{\mathrm{m}}$ & $\mathrm{k}_{\mathrm{d}}$ & $\mathbf{k}_{\mathrm{m}}$ & $\mathrm{k}_{\mathrm{d}}$ & $\mathbf{k}_{\mathrm{m}}$ \\
\hline \multirow{2}{*}{1.5} & 1.167 & 1.286 & 0.0046 & -0.0246 & 0.3391 & 0.1332 & 0.0209 & 0.0459 & 0.0121 & -0.0353 \\
\hline & 1.333 & 1.125 & 0.0015 & -0.0151 & 0.1665 & 0.0649 & 0.0115 & 0.0224 & 0.0033 & -0.0176 \\
\hline \multirow{2}{*}{2.0} & 1.333 & 1.500 & 0.0168 & -0.0374 & 0.4350 & 0.1531 & 0.0727 & 0.0779 & 0.0397 & -0.0507 \\
\hline & 1.667 & 1.200 & 0.0054 & -0.0228 & 0.2122 & 0.0730 & 0.0394 & 0.0371 & 0.0108 & -0.0258 \\
\hline \multirow{2}{*}{2.5} & 1.500 & 1.667 & 0.0302 & -0.0451 & 0.4655 & 0.1683 & 0.1257 & 0.1001 & 0.0647 & -0.0579 \\
\hline & 2.000 & 1.250 & 0.0096 & -0.0273 & 0.2266 & 0.0788 & 0.0674 & 0.0469 & 0.0174 & -0.0302 \\
\hline \multirow{2}{*}{3.0} & 1.667 & 1.800 & 0.0425 & -0.0502 & 0.4740 & 0.1801 & 0.1707 & 0.1162 & 0.0832 & -0.0616 \\
\hline & 2.333 & 1.286 & 0.0134 & -0.0304 & 0.2311 & 0.0833 & 0.0909 & 0.0537 & 0.0224 & -0.0329 \\
\hline \multirow{2}{*}{3.5} & 1.833 & 1.909 & 0.0531 & -0.0537 & 0.4744 & 0.1897 & 0.2074 & 0.1284 & 0.0963 & -0.0637 \\
\hline & 2.667 & 1.313 & 0.0165 & -0.0325 & 0.2319 & 0.0867 & 0.1099 & 0.0587 & 0.0260 & -0.0347 \\
\hline \multirow{2}{*}{4.0} & 2.000 & 2.000 & 0.0621 & -0.0563 & 0.4718 & 0.1975 & 0.2369 & 0.1378 & 0.1054 & -0.0650 \\
\hline & 3.000 & 1.333 & 0.0192 & -0.0341 & 0.2314 & 0.0895 & 0.1250 & 0.0624 & 0.0286 & -0.0360 \\
\hline
\end{tabular}

\begin{tabular}{|c|c|c|c|c|c|c|c|c|c|c|c|c|}
\hline \multirow{2}{*}{$a / b$} & \multicolumn{2}{|c|}{ Case XI } & \multicolumn{2}{|c|}{ Case XII } & \multicolumn{2}{|c|}{ Case XIII } & \multicolumn{2}{|c|}{ Case XIV } & \multicolumn{2}{|c|}{ Case XV } & \multicolumn{2}{|c|}{ Case XVI } \\
\hline & $k_{d}$ & $\mathrm{k}_{\mathrm{m}}$ & $\mathrm{k}_{\mathrm{d}}$ & $k_{m}$ & $k_{d}$ & $\mathrm{k}_{\mathrm{m}}$ & $\mathbf{k}_{\mathrm{d}}$ & $\mathbf{k}_{\mathrm{m}}$ & $k_{d}$ & $k_{m}$ & $\mathrm{k}_{\mathrm{d}}$ & $k_{m}$ \\
\hline 1.5 & 0.0064 & 0.0366 & 0.5186 & 0.2067 & 0.0249 & 0.0713 & 0.0241 & -0.0534 & 0.0424 & -0.0442 & 0.2281 & 0.0574 \\
\hline 2.0 & 0.0238 & 0.0675 & 0.6721 & 0.2469 & 0.0877 & 0.1256 & 0.0810 & -0.0757 & 0.0876 & -0.0597 & 0.3383 & 0.0926 \\
\hline 2.5 & 0.0435 & 0.0940 & 0.7213 & 0.2814 & 0.1530 & 0.1674 & 0.1333 & -0.0849 & 0.1188 & -0.0669 & 0.3995 & 0.1182 \\
\hline 3.0 & 0.0620 & 0.1171 & 0.7337 & 0.3114 & 0.2095 & 0.2009 & 0.1721 & 0.1122 & 0.1401 & -0.0707 & 0.4372 & 0.1384 \\
\hline 3.5 & 0.0783 & $\theta .1375$ & 0.7316 & 0.3379 & 0.2559 & 0.2559 & 0.1990 & 0.1426 & 0.1551 & 0.0821 & 0.4620 & 0.1552 \\
\hline 4.0 & 0.0923 & 0.1558 & 0.7238 & 0.3617 & 0.2938 & 0.2523 & 0.2172 & 0.1701 & 0.1660 & 0.0946 & 0.4794 & 0.1695 \\
\hline
\end{tabular}




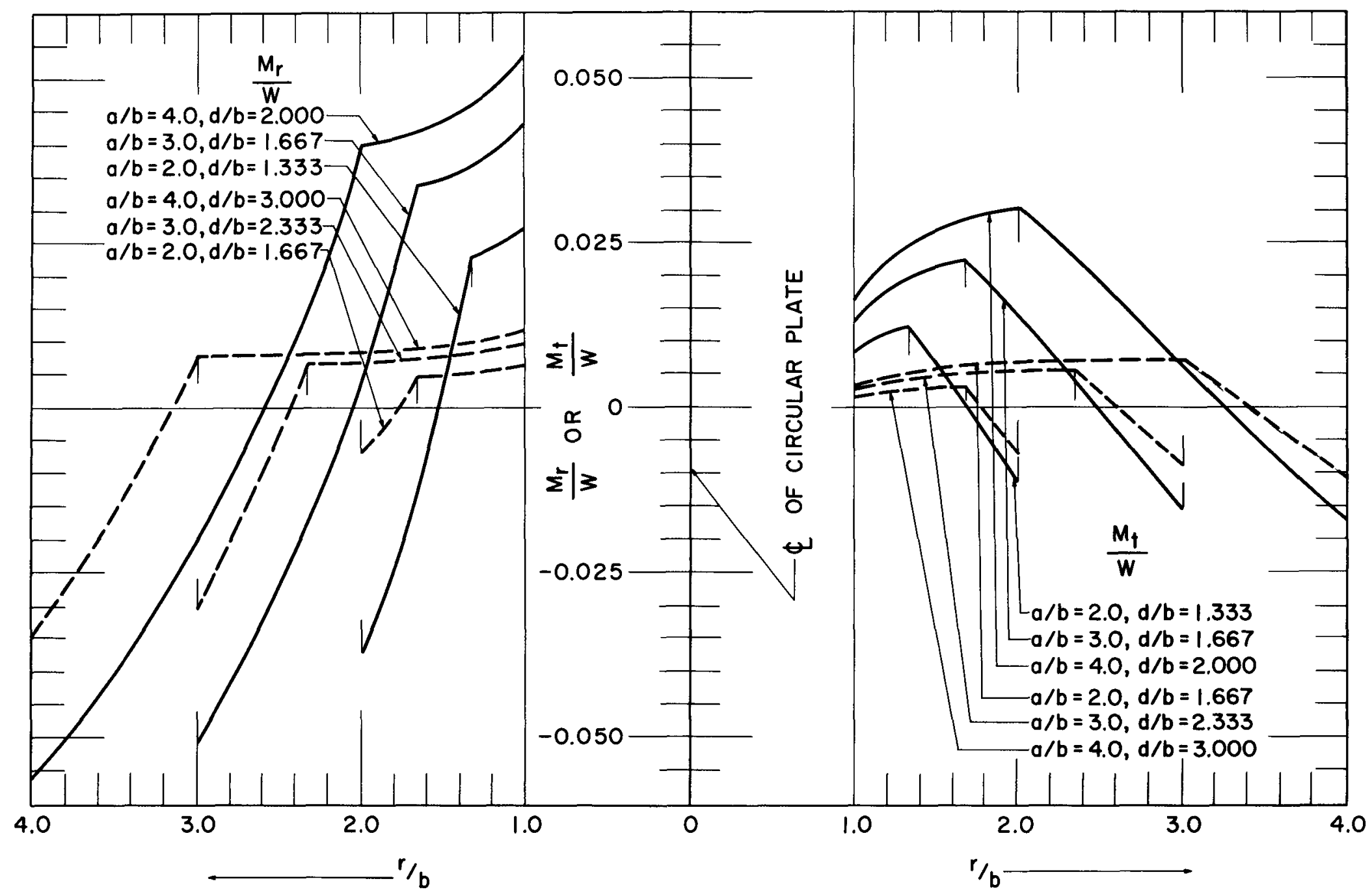

FIG. 2

RADIAL AND TANGENTIAL MOMENTS PER UNIFORM LOAD ON CONCENTRIC CIRCLE FOR CIRCULAR PLATE HAVING FIXED SUPPORTED OUTER EDGE

AND FIXED INNER EDGE

(CASE VIII, $\nu=0.3$ ) 


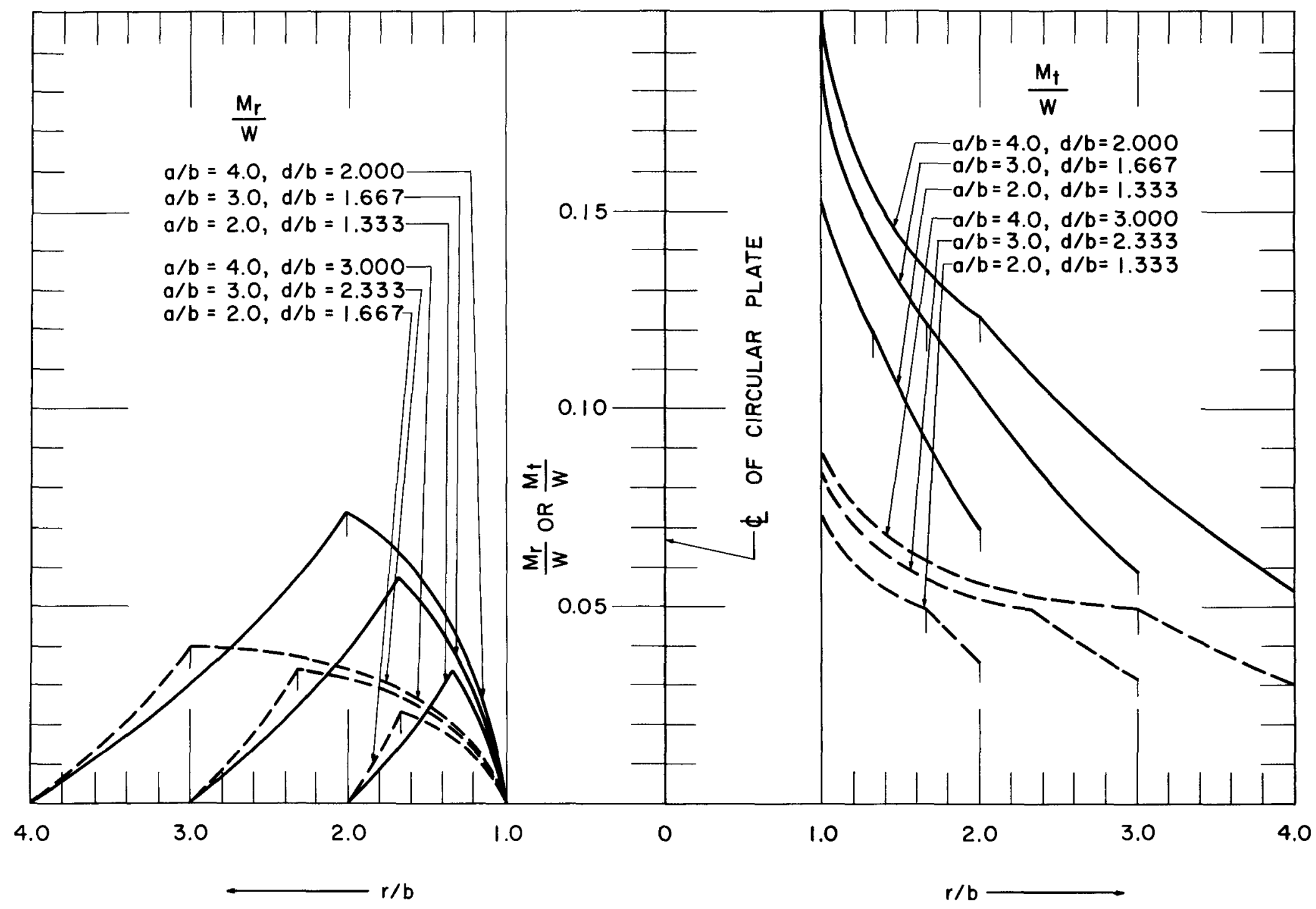

FIG. 3

RADIAL AND TANGENTIAL MOMENTS PER UNIFORM LOAD ON CONCENTRIC CIRCLE

FOR CIRCULAR PLATE HAVING SIMPLY SUPPORTED OUTER EDGE

AND FREE INNER EDGE

(CASE VIII, $\nu=0.3$ ) 


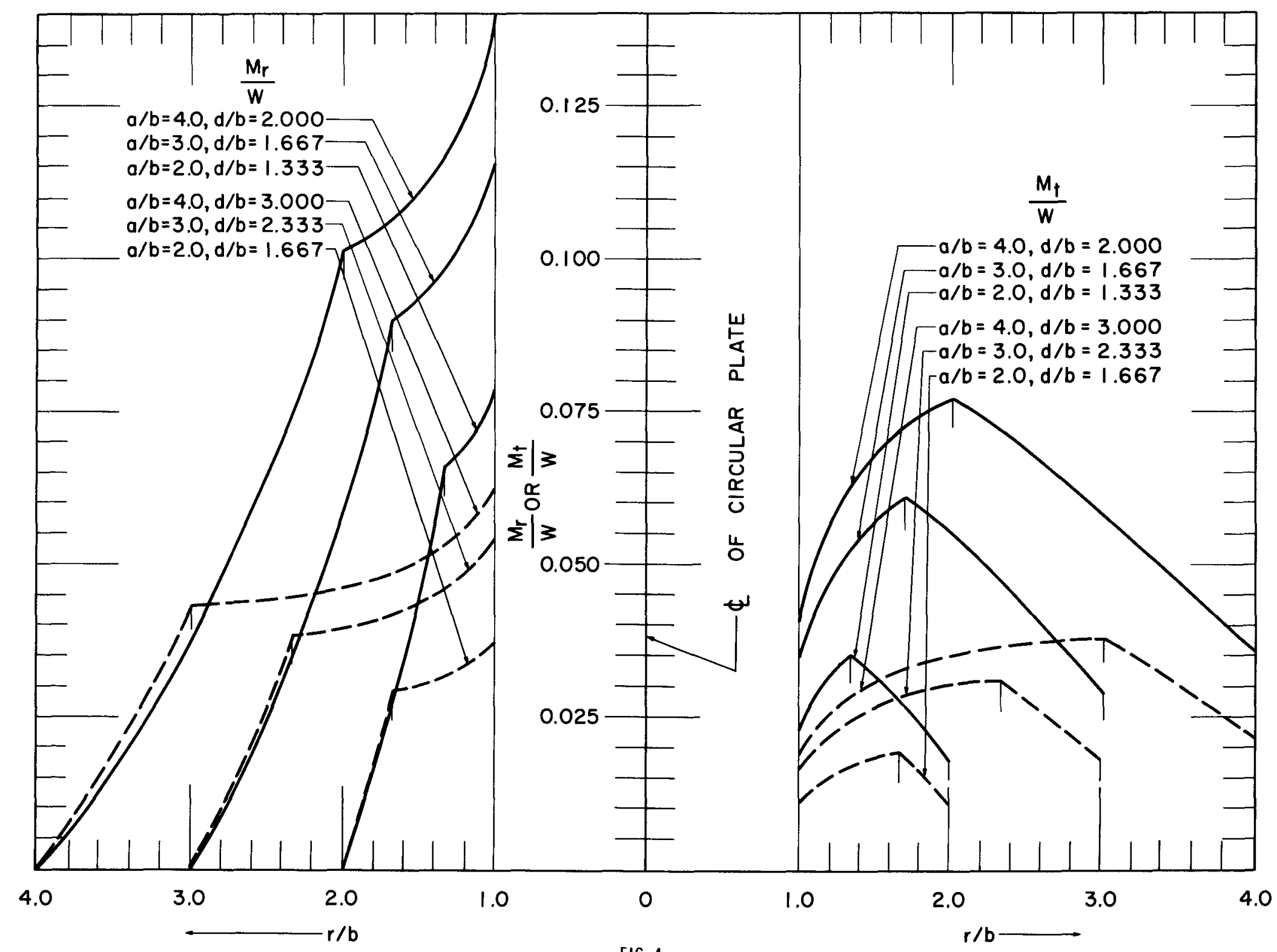

FIG. 4

RADIAL AND TANGENTIAL MOMENTS PER UNIFORM LOAD ON CONCENTRIC CIRCLE FOR CIRCULAR PLATE HAVING SIMPLY SUPPORTED OUTER EDGE

AND FIXED INNER EDGE

(CASE IX, $\nu=0.31$ 


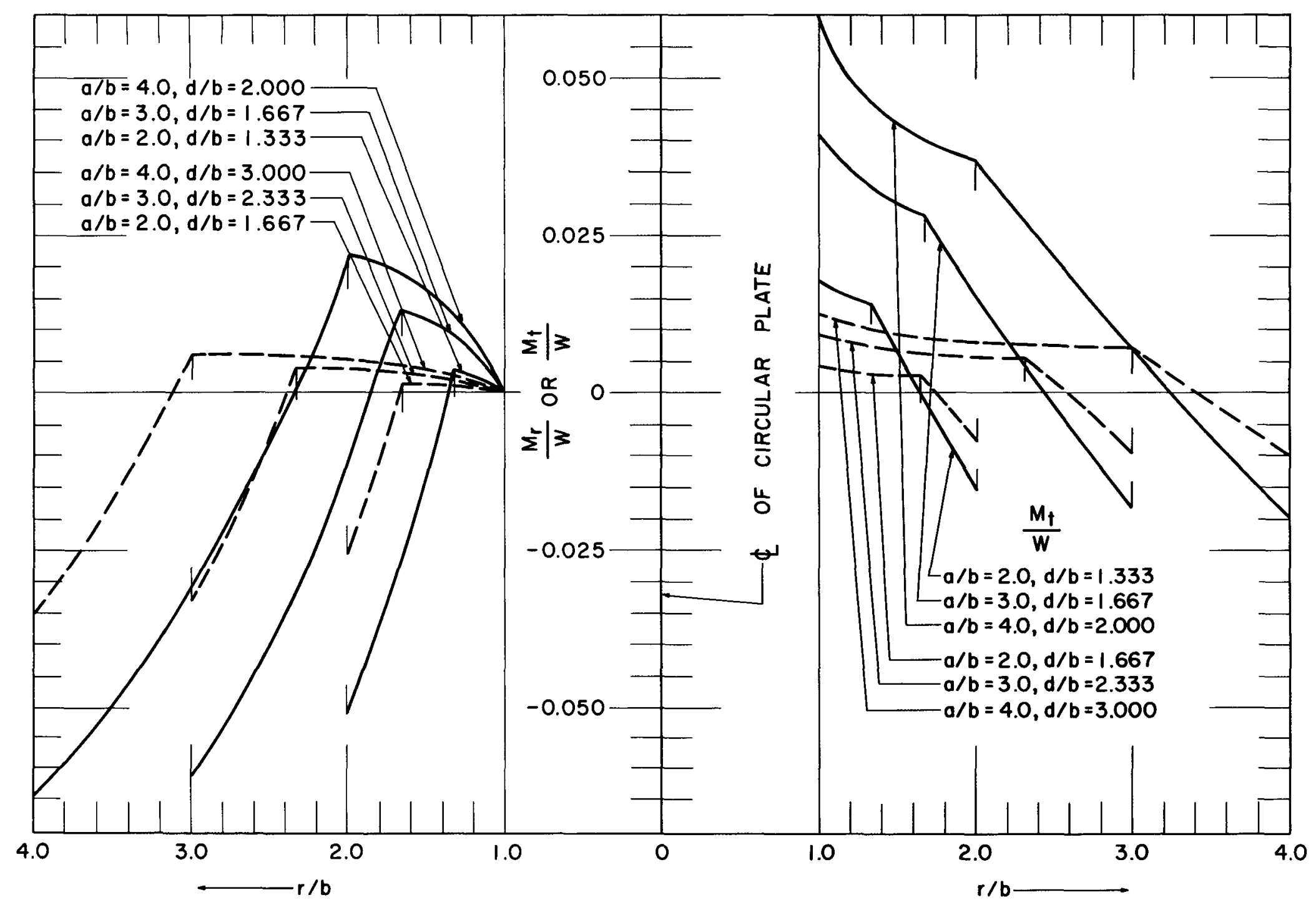

FIG. 5

RADIAL AND TANGENTIAL MOMENTS PER UNIFORM LOAD ON CONCENTRIC CIRCLE

FOR CIRCULAR PLATE HAVING FIXED SUPPORTED OUTER EDGE

AND FREE INNER EDGE

(CASE X, $\nu=0.3$ ) 


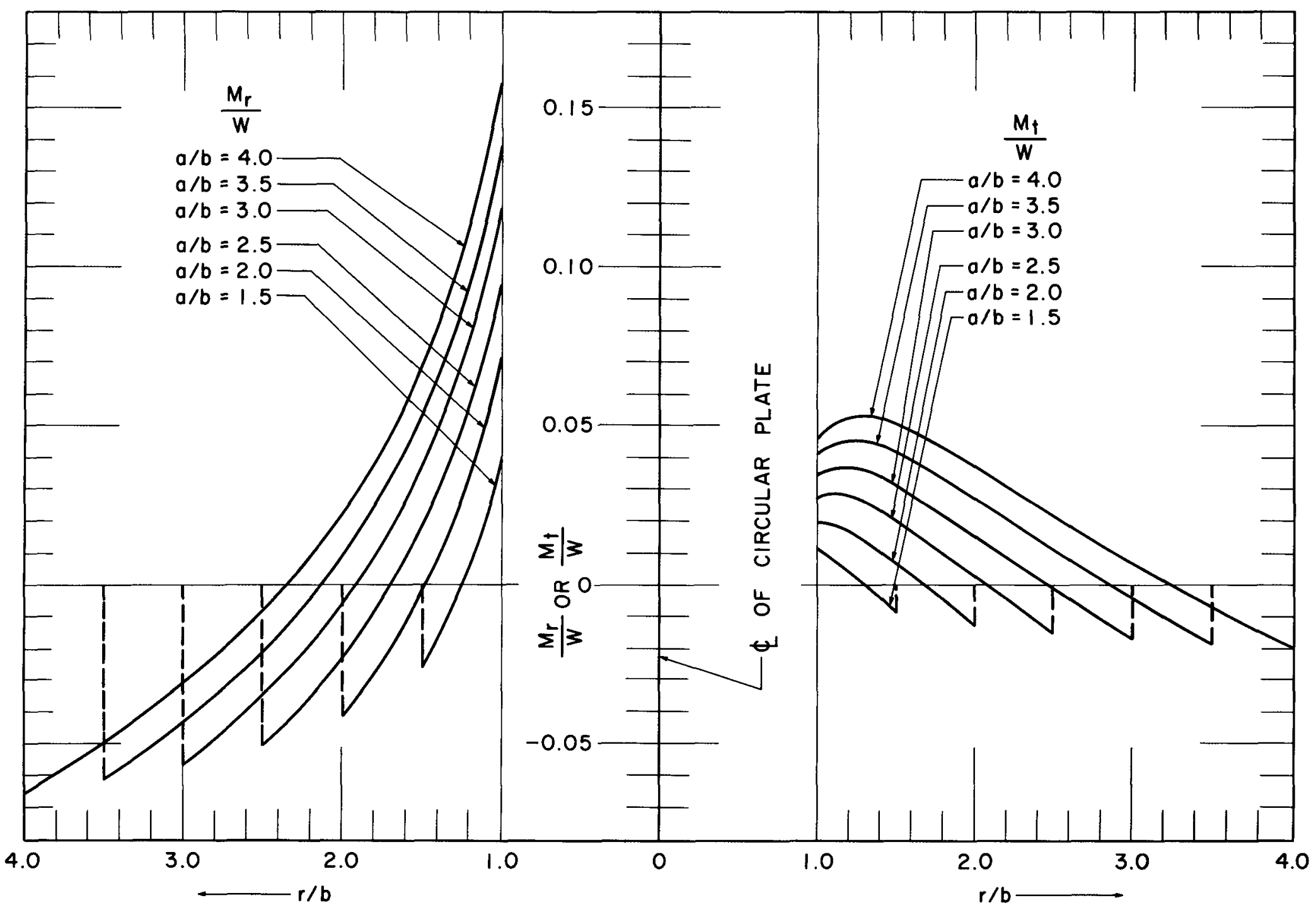

FIG. 6

RADIAL AND TANGENTIAL MOMENTS PER UNIFORM LOAD ON INNER CONCENTRIC CIRCLE FOR CIRCULAR PLATE HAVING FIXED SUPPORTED OUTER EDGE 


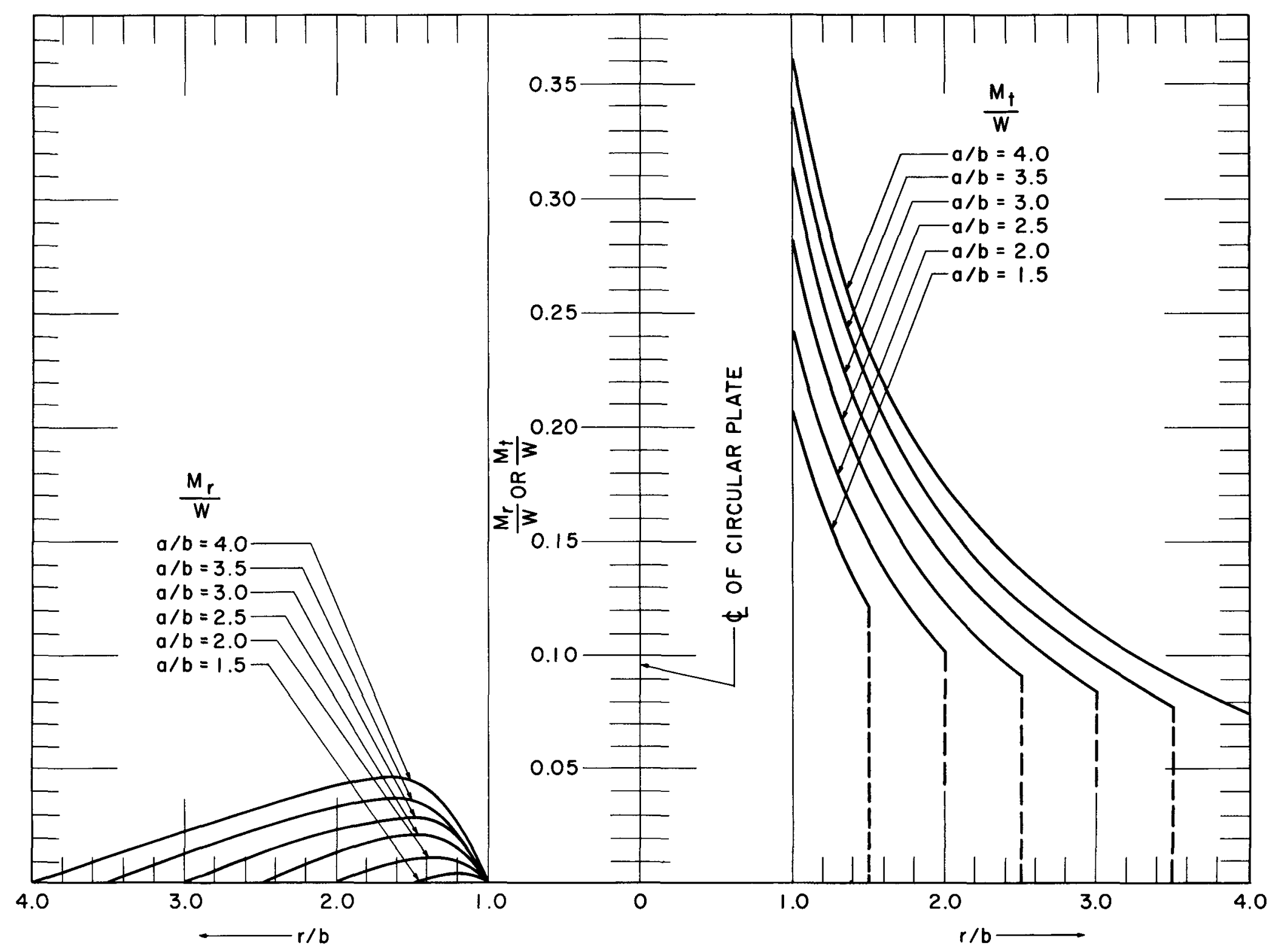

FIG. 7

RADIAL AND TANGENTIAL MOMENTS PER UNIFORM LOAD ON INNER CONCENTRIC CIRCLE FOR CIRCULAR PLATE HAVING SIMPLY SUPPORTED OUTER EDGE 


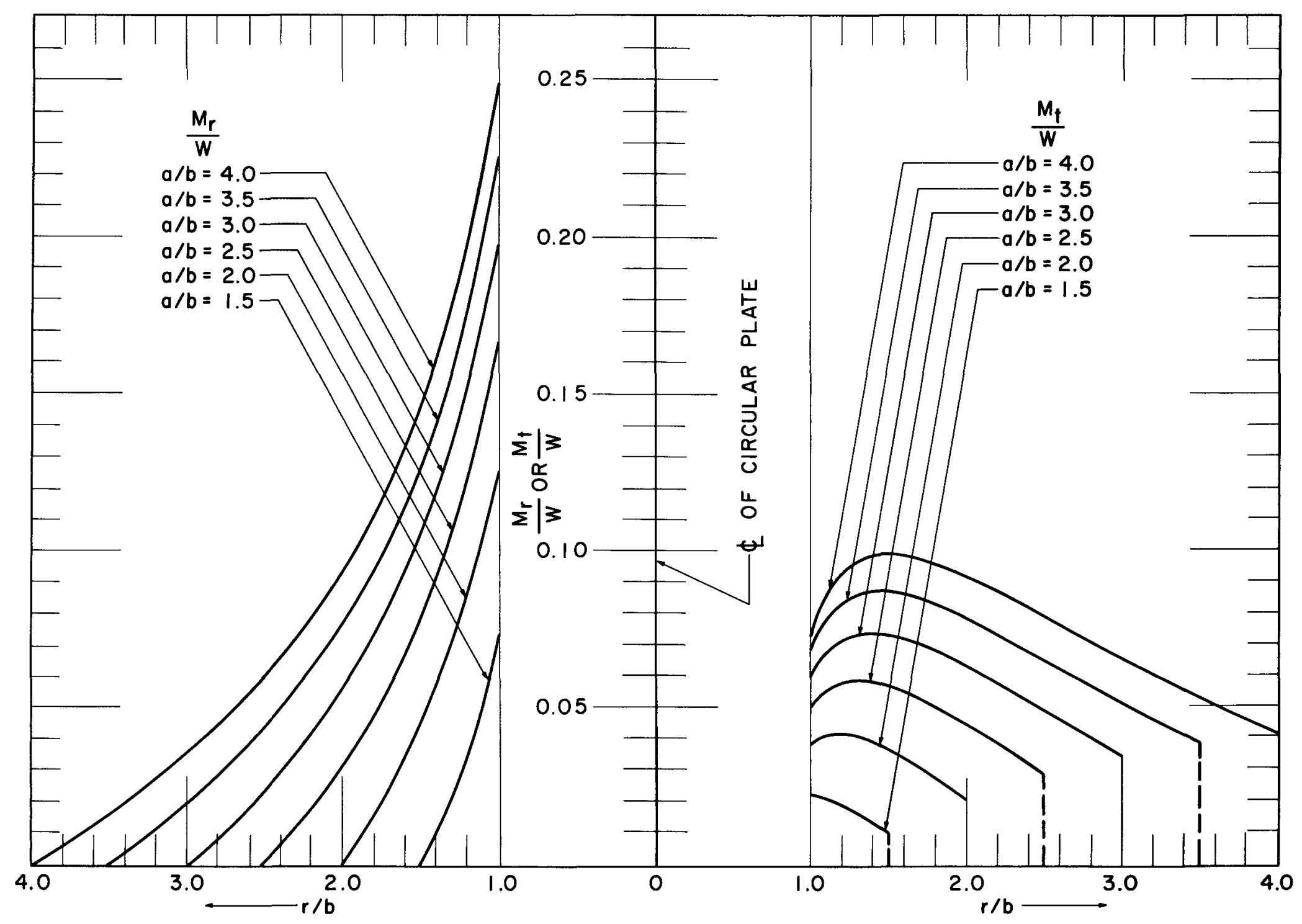

FIG. 8

RADIAL AND TANGENTI AL MOMENTS PER UNIFORM LOAD ON INNER CONCENTRIC CIRCLE FOR CIRCULAR PLATE HAVING SIMPLY SUPPORTED OUTER EDGE

AND FIXED INNER EDGE

(CASE XIII, $\nu=0.3$ ) 


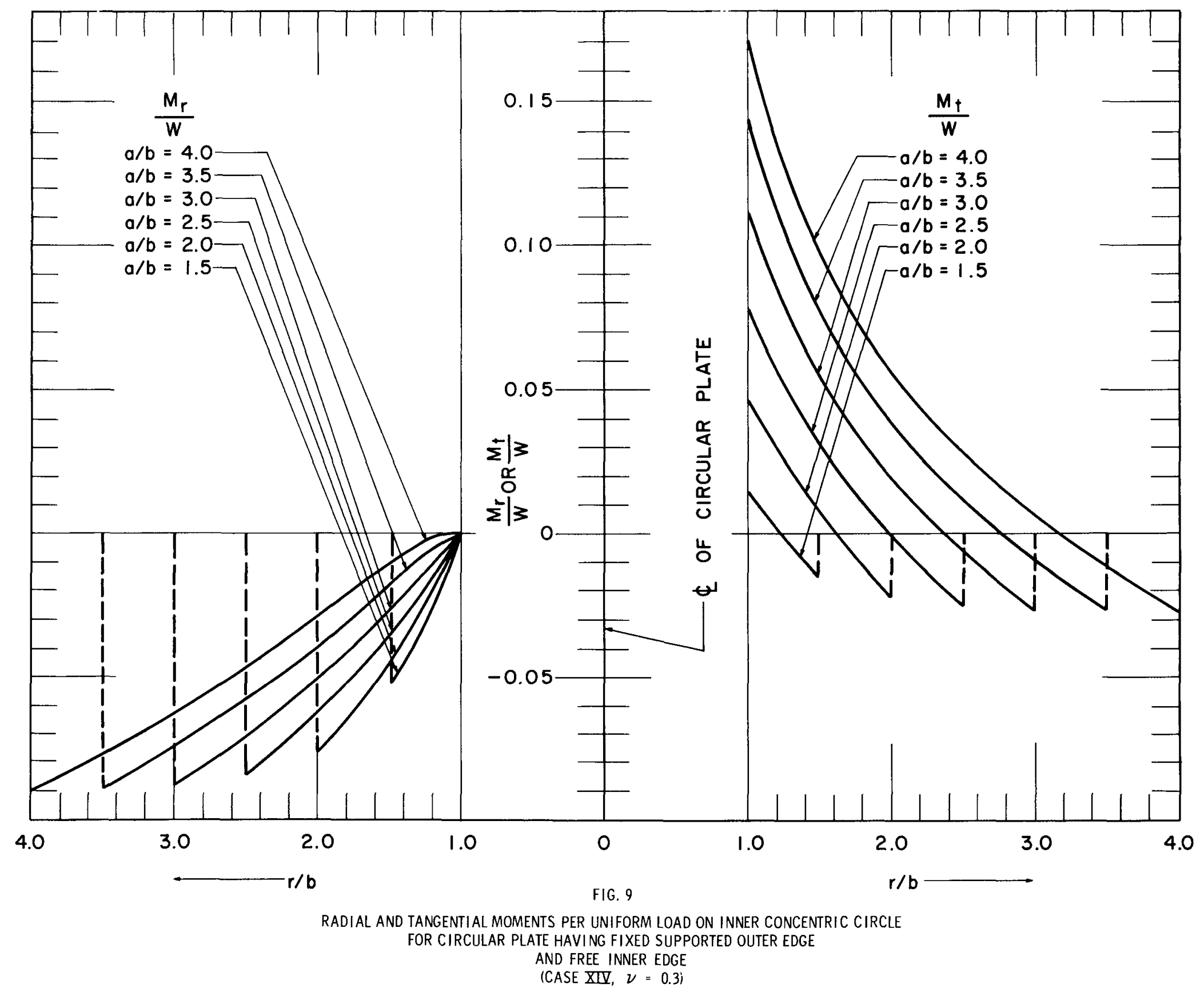




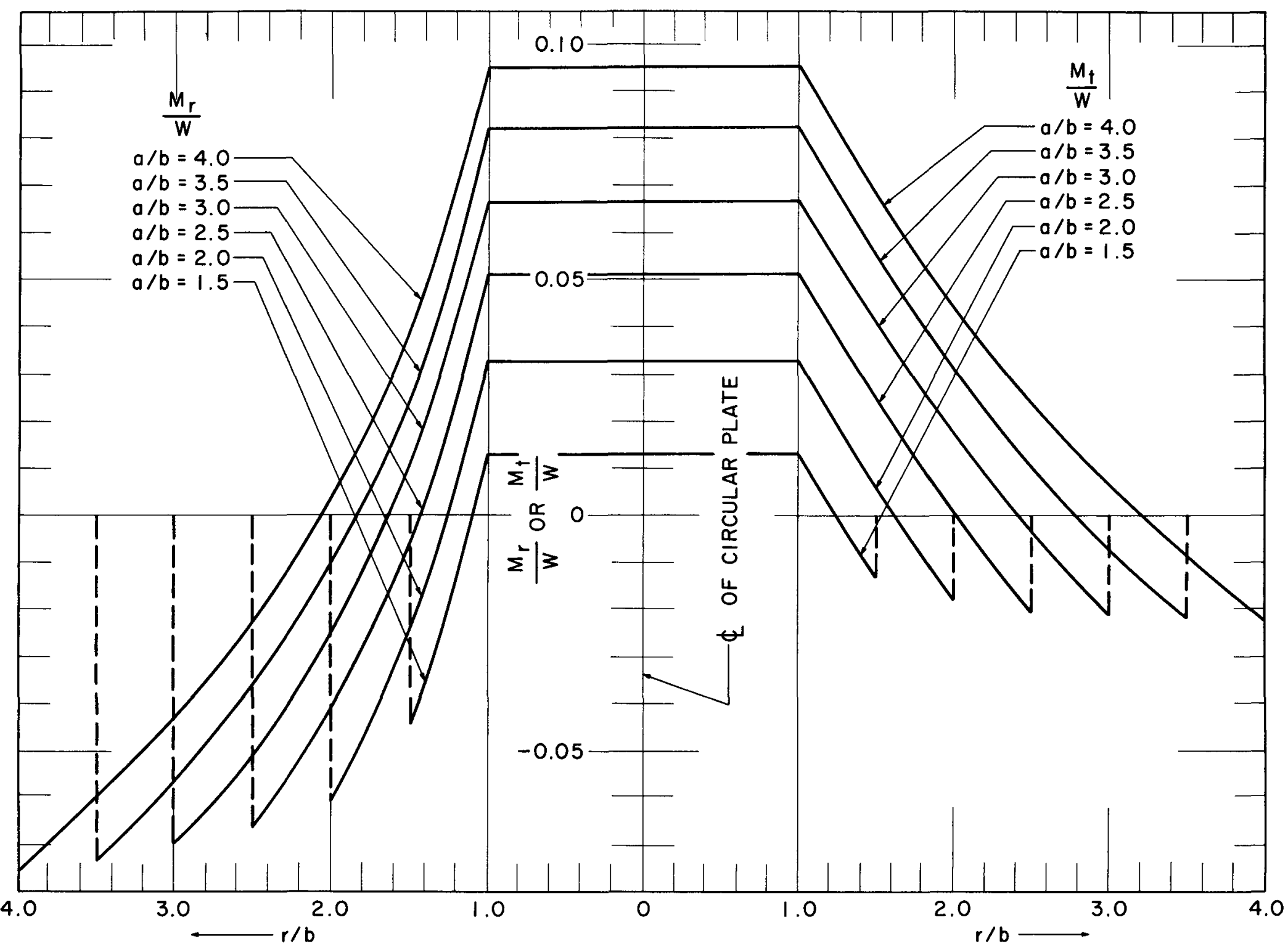

FIG. 10

RADIAL AND TANGENTIAL MOMENTS PER UNIFORM LOAD ON CONCENTRIC CIRCLE FOR SOLID CIRCULAR PLATE HAVING FIXED SUPPORTED OUTER EDGE (CASE XV, $\nu=0.3$ ) 


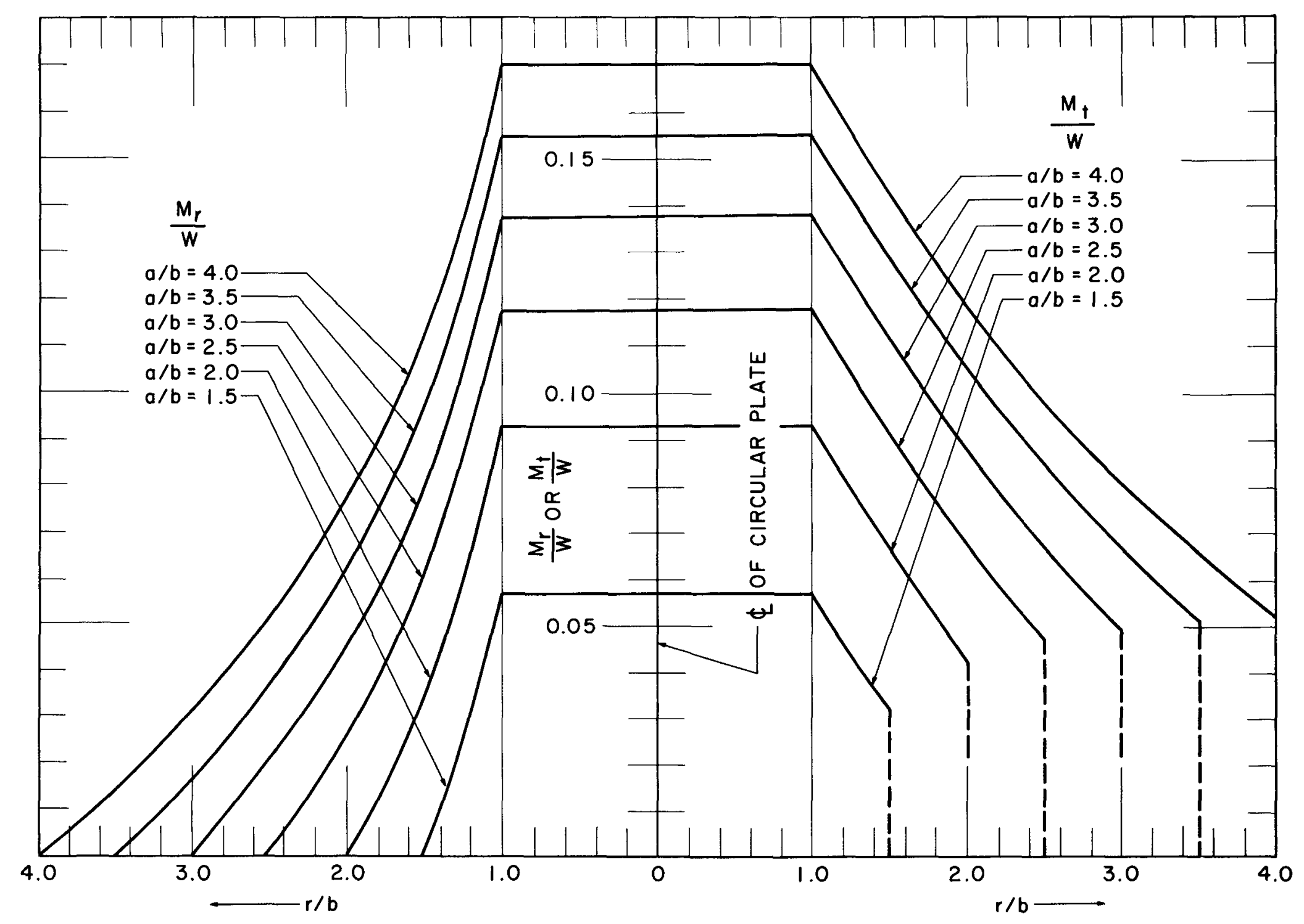

FIG. 11

RADIAL AND TANGENTIAL MOMENTS PER UNIFORM LOAD ON CONCENTRIC CIRCLE FOR SOLID CIRCULAR PLATE HAVING SIMPLY SUPPORTED OUTER EDGE (CASE XVI, $\nu=0.3$ ) 
The derived equations will now be applied to solving statically indeterminate, thin, flat, circular plates subjected to various loadings.

Numerical Example 1

The structural integrity of the copper diaphragm shown by Fig. 12 is to be determined. The following criteria apply: outer plate and load radius, $\mathrm{a}=4.00$ in.; inner plate and load radius, $\mathrm{b}=2.00$ in.; plate thickness, $h=0.125$ in.; constant force, $P=324$ lbf; maximum permissible unit stress, $\sigma_{\max }=6,0001 \mathrm{~b}_{\mathrm{f}} / \mathrm{in.}^{2}$; modulus of elasticity, $\mathrm{E}=15.0 \times 10^{6} \mathrm{lb} f$ in. $^{2}$; and Poisson's ratio, $\nu=0.33$.

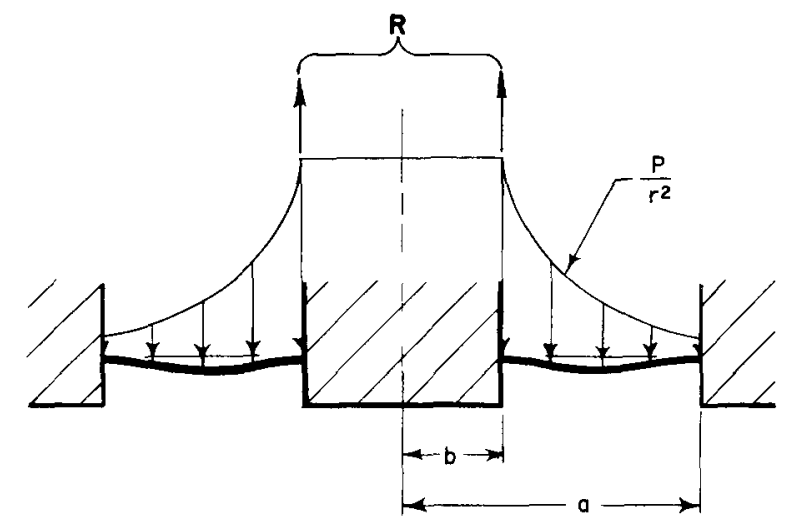

FIG. 12

STATICALLY INDETERMINATE DIAPHRAGM SUBJECTED TO A SYMMETRICAL VARIABLE LOAD

Since the required deflection formulas are known, the method of superposition is used to obtain the redundant reaction, $R$, at the inner radius. The deflection at the redundant support being zero, it is possible to write

$$
\Sigma w=0=w(\text { variable load })-w(\text { redundant load }) .
$$

If the maximum deflection formulas of Case I, Ref. 1, and Case XI of this paper are substituted in Eq. (11), the result is

$$
\begin{aligned}
R & =P \frac{\frac{b^{2}}{a^{2}}+\left(\ln \frac{a}{b}-1\right)\left[1-\frac{2 b^{2}}{a^{2}-b^{2}}\left(\ln \frac{a}{b}\right)^{2}\right]}{\frac{1}{\pi}\left[\frac{1}{2}\left(1-\frac{b^{2}}{a^{2}}\right)-\frac{2 b^{2}}{a^{2}-b^{2}}\left(\ln \frac{a}{b}\right)^{2}\right]} \\
& =\frac{k_{d} \text { (variable load) }}{k_{d} \text { (redundant load) } P,}
\end{aligned}
$$

where the $k_{d}$ 's are maximum deflection constants available from Refs. 1,3, 4, 5, and this paper, or are obtained from the program in this paper and 
in Ref. 1. Usage of $\mathrm{kd}$ from published tables is permissible in this case since the D's cancel and $\nu$ normally taken as 0.3 , is absent from remainder of the deflection equations. Using the aforementioned programs,

$$
R=324 \frac{0.05538}{0.02327}=771 \mathrm{lb} \text {. }
$$

The maximum bending moment for the redundant load occurs at the inner radius, while the maximum bending moment for the variable load occurs at the outer radius for $a / b=2.0$, per Ref. 1 . Therefore, moments at the inner radius and outer radius must be computed. By superposition, the moment expressions and numerical values at the inner radius and outer radius become, respectively,

$$
\begin{aligned}
\sum M_{r b}= & \frac{P}{4}\left\{1+\frac{2 a^{2}}{a^{2}-b^{2}}\left[\left(\ln \frac{a}{b}\right)^{2}-\ln \frac{a}{b}\right]\right\} \\
& -\frac{R}{2 \pi}\left[-\frac{1}{2}+\frac{a^{2}}{a^{2}-b^{2}} \ln \frac{a}{b}\right] ; \\
\Sigma M_{r b} & =-17.0 \text { in. }-1 b_{f} / \text { in.; }
\end{aligned}
$$

and

$$
\begin{aligned}
\Sigma M_{\text {ra }}= & \frac{P}{4}\left\{1+\frac{2 a^{2}}{a^{2}-b^{2}}\left[\frac{b^{2}}{a^{2}}\left(\ln \frac{a}{b}\right)^{2}-\ln \frac{a}{b}\right]\right\} \\
& -\frac{R}{2 \pi}\left[-\frac{1}{2}+\frac{b^{2}}{a^{2}-b^{2}} \ln \frac{a}{b}\right] \\
\Sigma M_{\text {ra }}= & 9.8 \text { in. }-1 b_{f} / \text { in. }
\end{aligned}
$$

From these computations, the maximum unit stress occurs at the inner radius; thus,

$$
\sigma_{r b}= \pm \frac{6 \Sigma M_{r b}}{h^{2}}= \pm \frac{6(-17.0)}{(0.125)^{2}}= \pm 6,530 \mathrm{lb}_{f} / \mathrm{in}^{2} .
$$

The imposed unit stress criterion has not been met; hence, the design must be revamped before the analysis is continued. 
Numerical Example 2

Determine the uniform plate thickness, the radial and tangential bending moment diagrams, the maximum unit bending stress of the symmetrically loaded, statically-indeterminate, circular aluminum plate illustrated by Fig. 13. Specifications are: outer plate and load radius, $\mathrm{a}=61.2 \mathrm{~cm}$; redundant support radius, $\mathrm{d}=40.8 \mathrm{~cm}$; inner plate and load radius, $b=24.0 \mathrm{~cm}$; maximum permissible deflection at inner plate radius, $\mathrm{w}_{\max }=0.08 \mathrm{~cm}$; variable load constant, $\mathrm{P}=147 \mathrm{~kg} f$; inner circular edge load, $W=1,430 \mathrm{~kg} ;$ modulus of elasticity, $E=70.3 \times 10^{4} \mathrm{~kg} f / \mathrm{cm}^{2}$; and Poisson's ratio, $\nu=0.3$.

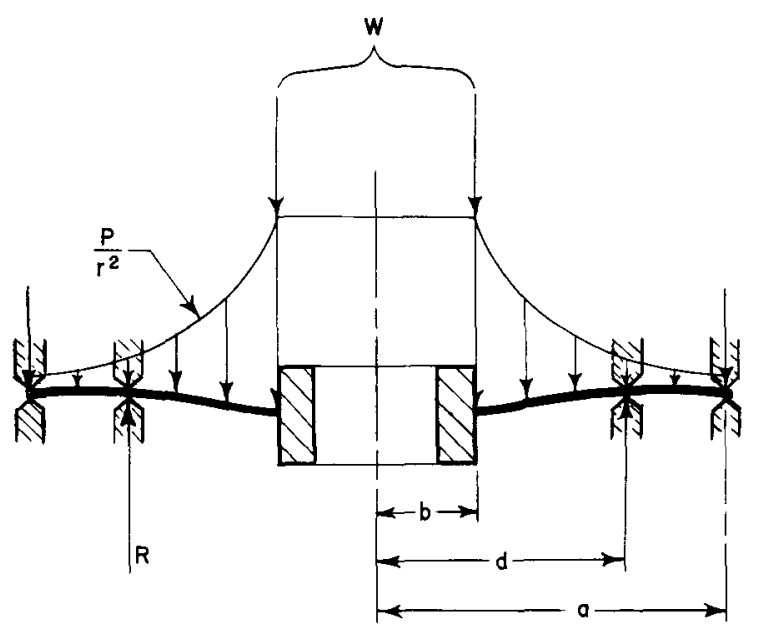

FIG. 13

STATICALLY INDETERMINATE CIRCULAR PLATE SUB JECTED TO SYMMETRICAL VARIABLF LOAD AND UNIFORM INNER EDGE LOAD

To determine the redundant reaction at radius $d$, the method of superposition is used again; i.e., the deflection at this support is zero. Mathematically,

$$
\begin{aligned}
\Sigma w_{d}=0= & w(\text { variable load })+w(\text { inner load })-w(\text { redundant load }) \\
= & \left.k_{d} \text { (variable load) } \frac{\mathrm{Pa}^{2}}{\mathrm{Eh}^{3}}+k_{d} \text { (inner load }\right) \frac{\mathrm{Wa}^{2}}{\mathrm{Eh}^{3}} \\
& \left.-\mathrm{k}_{\mathrm{d}} \text { (redundant load }\right) \frac{\mathrm{Ra}^{2}}{\mathrm{Eh}^{3}},
\end{aligned}
$$

where the $\mathrm{k}_{\mathrm{d}}$ 's are deflection constants at $\mathrm{d}$ to be determined. If a hand computer or the programs mentioned in Ref. 1 and this paper are used, the following constants are obtained from the appropriate equations of Cases III, XIII, and IX:

$$
\begin{aligned}
& \mathrm{k}_{\mathrm{d}}(\text { variable load })=0.45998 ; \mathrm{k}_{\mathrm{d}}(\text { inner load })=0.11126 ; \\
& \mathrm{k}_{\mathrm{d}}(\text { redundant load })=0.08287 ;
\end{aligned}
$$


where $\mathrm{a} / \mathrm{b}=2.55, \mathrm{a} / \mathrm{d}=1.5$, and $\mathrm{d} / \mathrm{b}=1.70$. Hence, Eq. (17) becomes

$$
0=0.45998(147)+0.11126(1430)-0.08287 R,
$$

from which,

$$
R=2736 \mathrm{~kg}_{\mathrm{f}} \text {. }
$$

The required plate thickness can now be determined; i.e.,

$$
\begin{aligned}
\Sigma \mathrm{w}_{\max }= & \mathrm{w}_{\max }(\text { variable load })+\mathrm{w}_{\max }(\text { inner load }) \\
& -\mathrm{w}_{\max }(\text { redundant load }) \\
= & \mathrm{k}_{\max }(\text { variable load }) \frac{\mathrm{Pa}^{2}}{\mathrm{Eh}^{3}}+\mathrm{k}_{\max } \text { (inner load) } \frac{\mathrm{Wa}^{2}}{\mathrm{Eh}^{3}} \\
& -\mathrm{k}_{\max }(\text { redundant load }) \frac{\mathrm{Ra}^{2}}{\mathrm{Eh}^{3}},
\end{aligned}
$$

where the $k_{\max }$ 's refer to the maximum deflection constants of the cases used. From the program results, or by hand computation,

$$
\begin{aligned}
& k_{\max }(\text { variable load })=0.63442 ; k_{\max }(\text { inner load })=0.15914 ; \\
& k_{\max }(\text { redundant load })=0.1126 .
\end{aligned}
$$

If the proper values are inserted in Eq. (21), and the equation is solved for plate thickness, the result is

$$
\begin{aligned}
h & =\left\{\frac{(61.2)^{2}}{0.08\left(70.3 \times 10^{4}\right)}[0.63442(147)+0.15914(1430)-0.11126(2736)]\right\}^{1 / 3} \\
& =[1.0937]^{1 / 3}=1.030 \mathrm{~cm} .
\end{aligned}
$$

viz.,

The radial and tangential moments are ascertained by superposition;

$$
\begin{aligned}
\sum M_{r} & \left.\left.\left.=M_{r} \text { (variable load }\right)+M_{r} \text { (inner load }\right)-M_{r} \text { (redundant load }\right) \\
& \left.\left.\left.=k_{r} \text { (variable load }\right) P+k_{r} \text { (inner load }\right) W-k_{r} \text { (redundant load }\right) R_{;} \\
\Sigma M_{t} & =M_{t}(\text { variable load })+M_{t}(\text { inner load })-M_{t}(\text { redundant load }) \\
& \left.\left.=k_{t} \text { (variable load }\right) P+k_{t}(\text { inner load }) W-k_{t} \text { (redundant load }\right) R .
\end{aligned}
$$

Figure 14 depicts the superpositioning of the moments. 


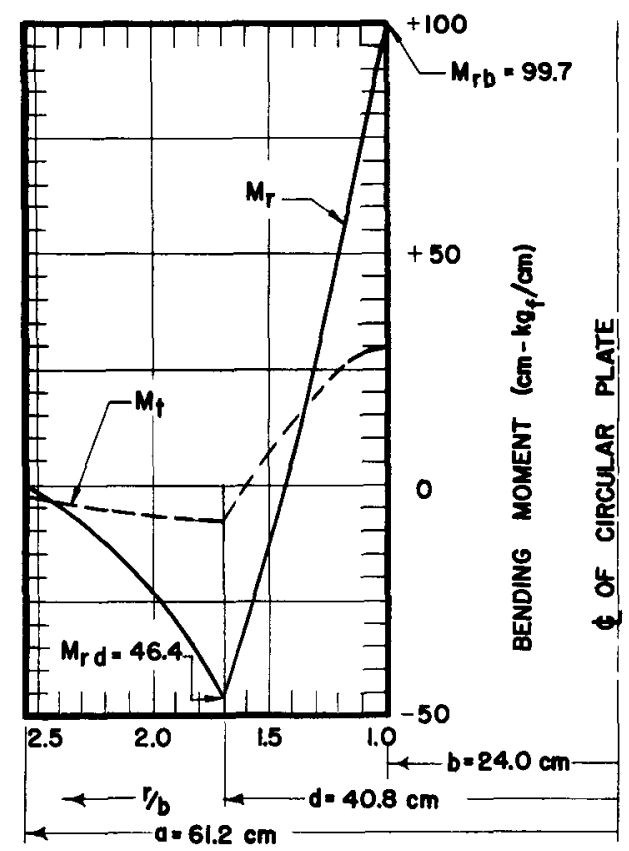

Fig. 14

BENDING MOMENT DIAGRAM, NUMERICAL EXAMPLE 2

From Fig. 14, the maximum unit stress at the inner radius becomes

$$
\sigma_{r b}= \pm \frac{6 \sum M_{r b}}{h^{2}}= \pm \frac{6(99.7)}{(1.030)^{2}}= \pm 564 \mathrm{~kg}_{f} / \mathrm{cm}^{2}
$$

\section{DISCUSSION OF GENERALIZED CASES}

Cases VIII and X of the four generalized cases are partially depicted in Ref. 5 as Cases 59 and 60. When the ascertained edge moment equations of Cases VIII and $X$ were converted to unit stress expressions, $\sigma_{t}=6 \mathrm{Mt}_{\mathrm{t}} / \mathrm{h}^{2}$ or $\sigma_{\mathrm{r}}=6 \mathrm{M}_{\mathrm{r}} / \mathrm{h}^{2}$, equations analogous to those in Ref. 5 were obtained, with the exception of signs. However, upon transformation, the maximum-deflection expressions did not concur. Because of these maximum-deflection discrepancies, extensive checking was performed.

The original derivations of the four generalized cases were attained by solving the moment equations and/or the integrated equations of the equilibrium equation with the imposed boundary and continuity conditions for the six unknown constants. Since agreement with Ref. 5 could not be achieved, these four cases were completely rechecked by the ANL Applied Mathematics Division to check the validity of the mathematics. As a further check, the author employed superposition by using derived slope and deflection formulas having uniform moments along the inner and outer edges in combination with one of the generalized cases to obtain another case.

The author of Ref. 5 has indicated that the deflection formulas of Cases 59 and 60 should be modified. Modifications will be incorporated in later editions of Ref. 5 . 


\section{ACKNOW LEDGMENT}

Grateful acknowledgment is extended to Joe Gvildys for his assistance in substantiating the author's derivations of the four generalized cases and for developing the moment-deflection program.

\section{REFERENCES}

1. Heap, J. C., Bending of Circular Plates under a Variable Symmetrical Load, ANL-6882 (April 1964).

2. Heap, J. C., Letters and Comment, The Metric System: Should We Convert?, Mech. Eng., Feb 1963, pp. 87-88.

3. Timoshenko, S., Strength of Materials, Part II, D. Van Nostrand Co., Inc., New York, Toronto, and London (1956), pp. 76-117.

4. Timoshenko, S., Theory of Plates and Shells, McGraw-Hill Book Co., Inc., New York and London (1940), pp. 34-84.

5. Roark, R. J., Formulas for Stress and Strain, McGraw-Hill Book Co., Inc., New York and London (1943), pp. 186-21 1. 